\title{
Composição florística de veredas no Município de Uberlândia, MG
}

\author{
GLEIN M. ARAÚJO', ${ }^{1,3}$, ANA A.A. BARBOSA ${ }^{1}$, \\ ADRIANA A. ARANTES ${ }^{1}$ e ALICE F. AMARAL ${ }^{2}$
}

(recebido: 12 de dezembro de 2001; aceito: 7 de agosto de 2002)

\begin{abstract}
Floristic composition of palm swamp communities near Uberlândia, Minas Gerais, Brazil). Over a two-year period, four palm swamp communities near Uberlândia, MG, Brazil, were studied for their floristic composition. Transects were randomly established to cross the following zones: palm swamp border (dryish soils), middle area (intermediate soil moisture), and lower zone (with permanently satured soils). The transects were censused each month and voucher herbarium specimens were collected and identified by means of keys, comparisons with identified material at HUFU, IBGE and UB, and with the help of specialists for some families. Voucher specimens were deposited at HUFU. A total of 526 species, 250 genera and 89 families were collected. The five families with highest number of species were: Poaceae (64), Asteraceae (63), Cyperaceae (54), Melastomataceae (27) e Fabaceae (23). In the palm swamp border, 361 species were collected, 168 of which were exclusive to this zone. A total of 300 species were collected in the middle zone, of which 75 were exclusive to this area. The lower zone had the lowest species diversity, with 52 exclusive species from a total of 136 taxa. The great richness found in the palm swamp possibly is due to soil differences, especially those related to humidity.
\end{abstract}

RESUMO - (Composição florística de veredas no Município de Uberlândia, MG). Foram estudadas quatro comunidades vegetais de veredas no Municipio de Uberlândia, MG, durante dois anos com o objetivo de conhecer sua composição florística. As veredas foram percorridas por meio de trilhas estabelecidas, ao acaso, passando pela borda (local de solo mais seco), meio (solo medianamente úmido) e fundo (solo saturado com água). A freqüência de visitas à cada vereda foi mensal e o material botânico coletado foi incluído no Herbarium Uberlandense (HUFU). A identificação das espécies foi feita utilizando-se chaves analíticas, consultas a especialistas de várias famílias e/ou comparação com exsicatas devidamente identificadas e depositadas nos herbários HUFU, IBGE e UB. No levantamento florístico foram registradas 526 espécies, 250 gêneros e 89 famílias. As cinco famílias com maior número de espécies foram Poaceae (64), Asteraceae (63), Cyperaceae (54), Melastomataceae (27) e Fabaceae (23). Na zona de borda da vereda foram amostradas 361 espécies sendo 168 exclusivas deste ambiente. Um total de 300 espécies ocorreu na zona de meio, sendo 75 exclusivas. Na zona de fundo ocorreu a menor riqueza específica, compreendendo um total de 136 espécies com 52 exclusivas desta área. A grande riqueza florística encontrada nas veredas amostradas deve-se, possivelmente, às diferenças edáficas, especialmente aquelas relacionadas com a umidade.

Key words - Cerrado biome, floristic composition, palm swamp, wetland

\section{Introdução}

As veredas são comunidades vegetais que ocorrem em áreas de nascentes na região do Brasil Central, tendo em sua periferia o cerrado (sentido amplo) (Eiten 1983, 1994). Estes ambientes são caracterizados principalmente, pela presença da palmeira Mauritia flexuosa L.f. (buriti) que ocorre, em geral, na parte mais alagada da vereda. A maior parte dessa comunidade é ocupada por uma densa vegetação herbácea, principalmente por espécies das famílias Cyperaceae, Eriocaulaceae e Poaceae e por um estrato arbustivo e subarbustivo de Melastomataceae e Rubiaceae

\footnotetext{
1. Universidade Federal de Uberlândia, Instituto de Biologia, Caixa Postal 593, 38400-902 Uberlândia, MG, Brasil.

2. Universidade Federal de Uberlândia, Curso de Mestrado em Ecologia e Conservação dos Recursos Naturais, Instituto de Biologia.

3. Autor para correspondência: glein@ufu.br.
}

(Magalhães 1966, Achá-Panoso 1978, Carvalho 1991). Diversos trabalhos sobre essa formação vegetal são representados por estudos de solos (Couto et al. 1985, Lima 1996, Guimarães 2001) ou topografia e evolução da paisagem (Lima 1996, Lima \& Queiroz Neto 1996). Com relação à vegetação, a maior parte dos trabalhos refere-se estritamente à descrição da paisagem sem detalhar a composição florística (Magalhães 1966, Boaventura 1978, Ferreira 1980, Carvalho 1991, Eiten 1994), que muitas vezes tem sido incluída juntamente com outras fisionomias similares às veredas, como brejo e campo úmido (Mendonça et al. 1998, Silva Júnior \& Felfili 1998, Felfili et al. 2001).

As veredas têm o seu papel reconhecido no equilíbrio geoecológico do bioma Cerrado, protegendo nascentes e fornecendo água, alimento e abrigo para a fauna silvestre (Castro 1980). No entanto, elas estão sendo degradadas devido à exploração de argila e turfa, atividade agropecuária, avanço da urbanização, construção de estradas e canais de drenagem. Em razão 
disso, as conseqüências têm sido desastrosas para este ambiente, com os assoreamentos, ressecamento dos solos, diminuição do volume hídrico, erosão e perda irreparável de sua beleza e biodiversidade (Guimarães 2001).

Este trabalho teve como objetivo realizar um levantamento florístico em quatro áreas de veredas no Município de Uberlândia, MG, contribuindo com informações básicas para futuros estudos da estrutura e dinâmica dessas comunidades vegetais.

\section{Material e métodos}

Áreas de estudo - As quatro veredas estudadas situam-se no Município de Uberlândia, Minas Gerais, Brasil (figura 1) e foram selecionadas em virtude de sua representatividade e do bom estado de conservação. A vereda 1 (figura 1 A) localiza-se a $15 \mathrm{~km}$ do centro da cidade de Uberlândia, na Reserva Vegetal do Clube Caça e Pesca Itororó (1860' S e $48^{\circ} 18^{\prime} \mathrm{W}$ ), ocupando área de 45 ha. A vereda 2 (figura 1 B), situa-se a $22 \mathrm{~km}$ do centro de Uberlândia, nas margens da estrada que leva a Campo Florido (19 $03^{\prime}$ 'S e $48^{\circ} 21^{\prime}$ ' W), com área de 8 ha. As veredas 3 e 4 (figura $1 \mathrm{C}$ ) localizam-se a $35 \mathrm{~km}$ do centro de Uberlândia, na Estação Ecológica do Panga (19 $11^{\prime} \mathrm{S}$ e $\left.48^{\circ} 24^{\prime} \mathrm{W}\right)$, tendo áreas, respectivamente, de 5 e 12 ha.

As veredas estudadas apresentam um denso estrato herbáceo-subarbustivo dominante, entremeado por pequenas árvores e a palmeira buriti. Nas quatro áreas o perfil topográfico apresenta-se em forma de vale aberto, tendo em sua periferia a vegetação de cerrado (sentido amplo). A zona de fundo de cada vereda mostrou variação quanto à umidade do solo, com sítios de drenagem difuso ou definido.

Amostragem florística - O levantamento florístico das quatro veredas foi realizado por meio de coletas de materiais botânicos, em estágio reprodutivo, ao longo de trilhas pré-estabelecidas e/ou de caminhadas aleatórias de forma a percorrer as áreas em toda sua extensão.

Cada uma das veredas foi subdividida em três zonas: a borda foi considerada a zona situada próximo ao cerrado, constituída por solo mais claro e com melhor drenagem; o meio, em solo mais escuro, saturado com água grande parte do ano e o fundo, em solo permanentemente saturado com água e essencialmente orgânico. Esta subdivisão foi baseada na zonação de solos de várzea do Estado de Minas Gerais sugerida pela Embrapa (1982) e por Almeida et al. (1983).

As coletas foram realizadas mensalmente por um período de dois anos (julho de 1998 a julho de 2000). A cada semana era visitada uma vereda para que se processassem as coletas. Para todo o material botânico coletado em estágio reprodutivo foram anotados os dados sobre hábito, zona de ocorrência e características morfológicas da planta.
O material coletado foi herborizado conforme os procedimentos usuais e posteriormente incorporado no acervo do Herbarium Uberlandense (HUFU), pertencente ao Instituto de Biologia da Universidade Federal de Uberlândia (UFU). As identificações foram feitas utilizando-se chaves analíticas, consultas a especialistas de várias famílias botânicas e/ou por comparação com exemplares devidamente identificados depositados nos herbários HUFU, IBGE (Instituto de Geografia e Estatística, em Brasília) e UB (Universidade de Brasília).

O sistema de classificação adotado foi o de Cronquist (1988), exceto para Pteridophyta que seguiu o sistema de Tryon \& Tryon (1982). Com base no material coletado durante o levantamento florístico foi elaborada uma listagem contendo as famílias, gêneros e espécies, com seus respectivos locais de ocorrência, hábito e número de registro no herbário HUFU.

A similaridade florística entre as veredas e suas respectivas zonas foi calculada ao nível de espécies. Para a análise de agrupamento foi utilizado o método (UPGMA) (Curi 1982) e o Índice de Sørensen, como distância de medida (Magurran 1988). Utilizou-se o programa PC-ORD, versão 2.0 (MacCune \& Mefford 1995), para a realização das análises.

\section{Resultados}

No estudo da composição florística das quatro veredas situadas no Município de Uberlândia foram encontradas 526 espécies, distribuídas em 250 gêneros e 89 famílias (tabela 1). Deste total, 63 famílias, 170 gêneros e 332 espécies foram representados pela classe Magnoliopsida (dicotiledôneas). A classe Liliopsida (monocotiledôneas) apresentou 21 famílias, 73 gêneros e 184 espécies. A divisão Pteridophyta contribuiu com cinco famílias, sete gêneros e nove espécies.

Nas veredas 1, 2, 3 e 4 foram amostradas, respectivamente, 307,266, 218 e 242 espécies pertencentes a 62, 64, 53 e 54 famílias botânicas. As cinco famílias que se destacaram em riqueza de espécies, foram: Poaceae (64 espécies), Asteraceae (63), Cyperaceae (54), Melastomataceae (27) e Fabaceae (23). Essas famílias representam 43,9\% do total de espécies coletadas. Outras 41 famílias representadas por uma espécie acumularam apenas $7,8 \%$ da riqueza florística. Destas, 25 famílias foram exclusivas de ambientes mais úmidos das veredas (na zona do meio e fundo) (tabela 1).

As plantas com hábito herbáceo e subarbustivo predominaram nas veredas estudadas, com 278 espécies herbáceas $(52,8 \%), 137$ subarbustivas $(26 \%), 51$ arbustivas (9,7\%), 22 arbóreas $(4,2 \%)$ e as demais $7,3 \%$ 


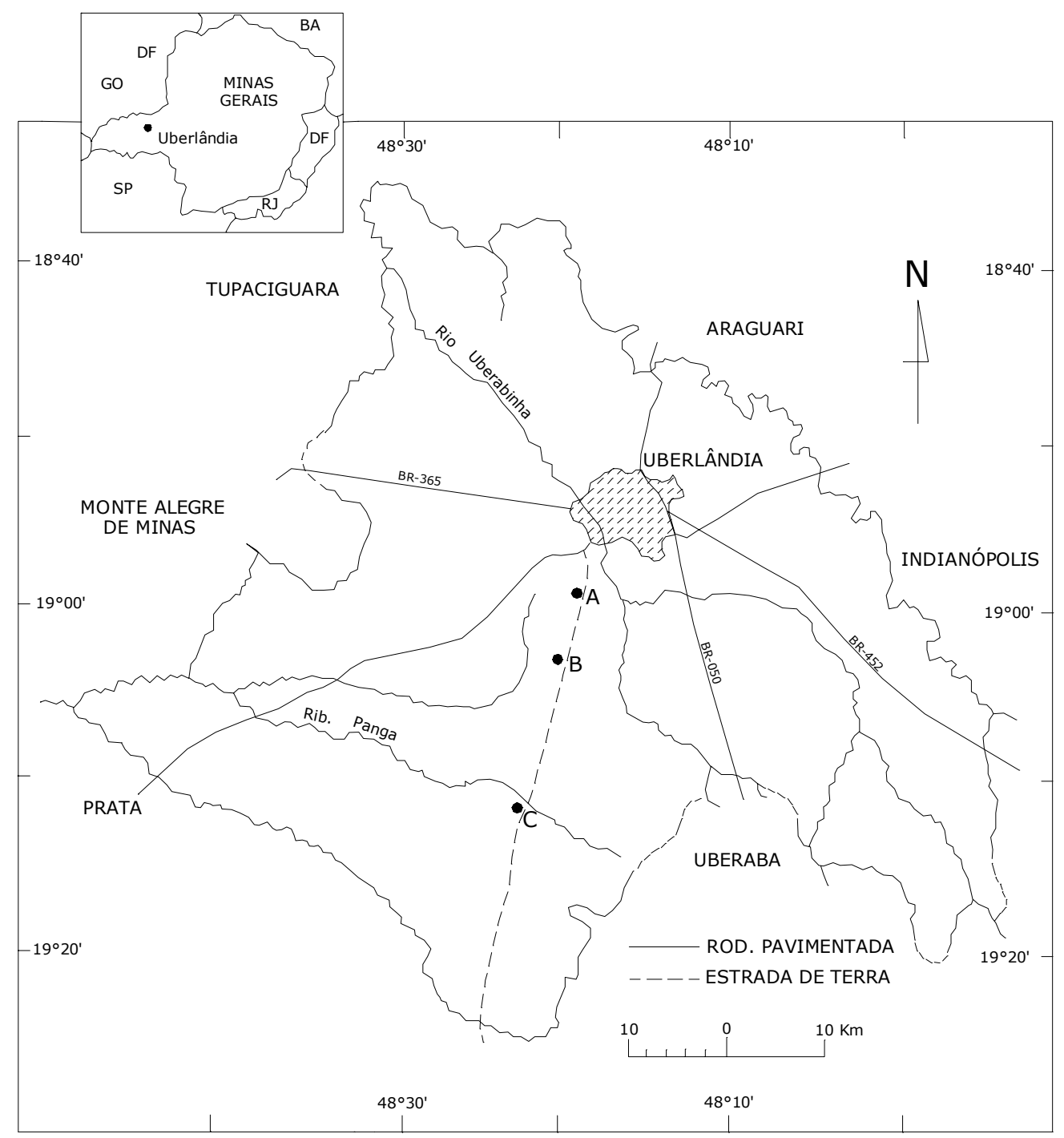

Figura 1. Localização do Município de Uberlândia, MG, e das veredas amostradas: A- Reserva Vegetal do CCPIU, vereda 1; B - Estrada para Campo Florido, vereda 2; C - Estação Ecológica do Panga, veredas 3 e 4.

(tabela 1). A proporção do número de espécies do componente herbáceo-subarbustivo em relação ao arbustivo-arbóreo foi de aproximadamente 6:1.

A análise de agrupamento das espécies coletadas nas quatro veredas mostrou a formação de três grupos florísticos bem distintos, independentes da localização das veredas. As espécies coletadas nas zonas de borda e meio formaram dois grupos mais similares entre si, com percentuais de similaridade acima de 50\%, enquanto que a zona de fundo mostrou percentual de similaridade entre $20 \%$ a $50 \%$ (figura 2 ).

Com relação à zonação das veredas, a borda foi a mais rica com 361 espécies ( $82 \%$ herbáceas e subarbustivas), sendo que $46,5 \%$ do total foram exclusivas deste ambiente. Nessa zona, próximo ao cerrado, predominam espécies das famílias Asteraceae, Caesalpiniaceae, Fabaceae, Melastomataceae, Myrtaceae e Rubiaceae. Miconia albicans (Melastomataceae), Styrax ferrugineus (Styracaceae), Vochysia tucanorum (Vochysiaceae), Myrcia tomentosa e Blepharocalyx salicifolius (Myrtaceae) foram algumas das espécies com hábito arbóreo que ocorreram nessa área. Dentre as herbáceas nativas, as gramíneas Echinolaena inflexa e Loudetiopsis chrysothrix ocorreram com maior densidade nesse ambiente junto com espécies exóticas como Brachiaria decumbens (brachiaria) e Melinis minutiflora (capim-gordura) (tabela 1).

Na zona de meio foram encontradas 300 espécies, 
Tabela 1. Espécies em ordem de família coletadas nas veredas do Município de Uberlândia, Minas Gerais. Veredas estudadas $=1$, 2, 3, 4. Hábito, Arv = árvore, Arb = arbusto, $\mathrm{Sub}=$ subarbusto, Erv = erva, Lia = liana, Par = parasita. Zona de ocorrência, B = borda, $\mathrm{M}=$ meio, $\mathrm{F}$ = fundo. $\mathrm{N}$ = número do registro no herbário (HUFU).

\begin{tabular}{|c|c|c|c|c|c|c|}
\hline Famílias / espécies & Veredas & Hábito & $\mathrm{B}$ & M & $\mathrm{F}$ & $\mathrm{N}$ \\
\hline \multicolumn{7}{|l|}{ ACANTHACEAE } \\
\hline Justicia polygaloides (S. Mor.) Lindau & $1,3,4$ & Sub & $\mathrm{x}$ & $\mathrm{x}$ & & 22286 \\
\hline Ruellia dissitifolia (Ness) Hiern. & 1,2 & Erv & $\mathrm{x}$ & & & 16992 \\
\hline Ruellia cf. nitens (Nees) Wassh. & 1 & Sub & $\mathrm{x}$ & & & 20094 \\
\hline Stenandrium hirsutum Nees & $1,2,3,4$ & Erv & $\mathrm{x}$ & $\mathrm{x}$ & & 17817 \\
\hline \multicolumn{7}{|l|}{ ALISMATACEAE } \\
\hline Echinodorus latifolius (Seub.) Rataj & 2,3 & Erv & & $\mathrm{x}$ & & 18111 \\
\hline Echinodorus longipetalus Micheli & 2 & Erv & & & $\mathrm{x}$ & 18776 \\
\hline Sagittaria rhombifolia Cham. & 1 & Erv & & & $\mathrm{x}$ & 21700 \\
\hline \multicolumn{7}{|l|}{ AMARANTHACEAE } \\
\hline Pfaffia jubata Mart. & 1 & Sub & $\mathrm{x}$ & & & 20348 \\
\hline Pfaffia prostata Mart. & $1,2,3$ & Sub & $\mathrm{x}$ & & & 21773 \\
\hline \multicolumn{7}{|l|}{ ANACARDIACEAE } \\
\hline Tapirira guianensis Aubl. & 4 & Arv & $\mathrm{x}$ & & & 18233 \\
\hline \multicolumn{7}{|l|}{ ANNONACEAE } \\
\hline Xylopia aromatica (Lam.) Mart. & 4 & Arv & $\mathrm{x}$ & & & 21547 \\
\hline \multicolumn{7}{|l|}{ APIACEAE } \\
\hline Apium leptophyllum (Pers.) F. Muell. & 2 & Erv & $\mathrm{x}$ & & & 18112 \\
\hline Eryngium cf. junceum Cham. \& Schlecht. & 1 & Erv & $\mathrm{x}$ & & & 18304 \\
\hline Eryngium ebracteatum Lam. & 1,2 & Erv & $\mathrm{x}$ & $\mathrm{x}$ & & 21138 \\
\hline Eryngium elegans Cham. \& Schlecht. & 1,2 & Erv & $\mathrm{x}$ & & & 21791 \\
\hline \multicolumn{7}{|l|}{ APOCYNACEAE } \\
\hline Odontadenia hypoglauca (Stand.) M. Arg. & $1,2,3$ & Lia & & $\mathrm{x}$ & $\mathrm{x}$ & 21297 \\
\hline Odontadenia lutea (Vell.) Marckgraf & 1 & Lia & & $\mathrm{x}$ & & 21176 \\
\hline Odontadenia sp. & 3 & Lia & & $\mathrm{x}$ & & 20845 \\
\hline Rhabdadenia pohlii M. Arg. & 1 & Lia & & $\mathrm{x}$ & & 21526 \\
\hline \multicolumn{7}{|l|}{ AQUIFOLIACEAE } \\
\hline Ilex affinis Gardn. & 1 & Arv & & & $\mathrm{x}$ & 21056 \\
\hline \multicolumn{7}{|l|}{ ARACEAE } \\
\hline Caladium sp. & 1,3 & Ver & & $\mathrm{x}$ & $\mathrm{x}$ & 18584 \\
\hline Montrichardia cf. linifera Schott & 1,2 & Ver & & $\mathrm{x}$ & $\mathrm{x}$ & 21180 \\
\hline Xanthosoma striatipes (Kunth) Madison & 1,4 & Ver & & $\mathrm{x}$ & $\mathrm{x}$ & 19883 \\
\hline \multicolumn{7}{|l|}{ ARECACEAE } \\
\hline Mauritia flexuosa L.f. & $1,2,3,4$ & Arb & & & $\mathrm{x}$ & 20719 \\
\hline \multicolumn{7}{|l|}{ ASCLEPIADACEAE } \\
\hline Barjonia harleyi Fontella \& Marquete & 1 & Sub & & $\mathrm{x}$ & & 18884 \\
\hline Blepharodon bicuspidatus E. Fourn. & 4 & Lia & $\mathrm{x}$ & & & 18586 \\
\hline Melinia sp. & 2,4 & Lia & & $\mathrm{x}$ & $\mathrm{x}$ & 21656 \\
\hline Oxypetalum regnellii (Malme) Malme & 1,3 & Lia & & $\mathrm{x}$ & $\mathrm{x}$ & 21477 \\
\hline Schulbertia grandiflora Mart. (Turcuz.) Fontella & 4 & Lia & & $\mathrm{x}$ & $\mathrm{x}$ & 22813 \\
\hline \multicolumn{7}{|l|}{ ASTERACEAE } \\
\hline Acanthospermum australe (Loefl.) O. Kuntze & 4 & Ver & $\mathrm{x}$ & & & 19712 \\
\hline Achyrocline alata DC. & $1,2,3,4$ & Ver & $\mathrm{x}$ & $\mathrm{x}$ & & 20666 \\
\hline Achyrocline satureoides (Lam.) DC. & $2,3,4$ & Ver & $\mathrm{x}$ & $\mathrm{x}$ & & 19720 \\
\hline Adenostemma suffruticosum (Gard.) King \& H. Rob. & 3 & Sub & $\mathrm{x}$ & $\mathrm{x}$ & & 21139 \\
\hline Ageratum conyzoides L. & 2,3 & Ver & & $\mathrm{x}$ & $\mathrm{x}$ & 20142 \\
\hline Ageratum fastigiatum (Gard.) King \& H. Rob. & $1,2,3,4$ & Sub & $\mathrm{x}$ & $\mathrm{x}$ & & 20083 \\
\hline Baccharis dracunculifolia DC. & $1,3,4$ & Arb & $\mathrm{x}$ & $\mathrm{x}$ & $\mathrm{x}$ & 23203 \\
\hline Baccharis humilis Sch. Bip. ex Baker & 1,4 & Ver & $\mathrm{x}$ & $\mathrm{x}$ & & 22702 \\
\hline
\end{tabular}


(cont.)

\begin{tabular}{|c|c|c|c|c|c|c|}
\hline Famílias / espécies & Veredas & Hábito & $\mathrm{B}$ & $\mathrm{M}$ & $\mathrm{F}$ & $\mathrm{N}$ \\
\hline \multicolumn{7}{|l|}{ ASTERACEAE } \\
\hline Baccharis lymanii G.M. Barroso & 2,3 & Arb & $\mathrm{x}$ & $\mathrm{x}$ & & 20886 \\
\hline Baccharis subdentata DC. & 3,4 & Ver & $\mathrm{x}$ & & & 20140 \\
\hline Baccharis trimera DC. & 3 & Arb & & & $\mathrm{X}$ & 23205 \\
\hline Baccharis varians DC. & 4 & Arb & $\mathrm{x}$ & $\mathrm{x}$ & & 23468 \\
\hline Chaptalia integrifolia Baker & 1 & Ver & & $\mathrm{x}$ & & 21421 \\
\hline Coniza canadensis (L.) Cronquist & 3,4 & Ver & $\mathrm{x}$ & $\mathrm{x}$ & $\mathrm{X}$ & 21735 \\
\hline Coniza sp. & 1 & Ver & $\mathrm{x}$ & & & 20392 \\
\hline Elephantopus biflorus (Less.) Sch. Bip. & $1,3,4$ & Ver & $\mathrm{x}$ & & & 20704 \\
\hline Elephantopus palustris Gardn. & 2 & Ver & & $\mathrm{x}$ & & 20720 \\
\hline Elephantopus riparius Gardn. & 2 & Ver & $\mathrm{x}$ & & & 19709 \\
\hline Erechtites hieraciifolia (L.) Raf. ex DC. & $1,2,3,4$ & Ver & $\mathrm{x}$ & $\mathrm{X}$ & & 17831 \\
\hline Erechtites sp. & 1,4 & Erv & & $\mathrm{x}$ & $\mathrm{x}$ & 21731 \\
\hline Erigeron maximus (D. Don.) DC. & 2,4 & Erv & & & $\mathrm{x}$ & 19868 \\
\hline Eupatorium aff. grande Sch. Bip. ex Baker & 2,4 & Arb & $\mathrm{x}$ & $\mathrm{x}$ & & 22499 \\
\hline Eupatorium amygdalinum Lam. & 3 & Sub & & $\mathrm{x}$ & & 17282 \\
\hline Eupatorium barbacence Hieron. & 1,4 & Sub & $\mathrm{x}$ & $\mathrm{x}$ & & 22749 \\
\hline Eupatorium basifolium Malme & 4 & Sub & $\mathrm{x}$ & $\mathrm{x}$ & & 18388 \\
\hline Eupatorium bupleurifolium (DC.) Baker & 2 & Sub & & $\mathrm{x}$ & $\mathrm{x}$ & 18116 \\
\hline Eupatorium clematideum Griseb. & 1 & Sub & $\mathrm{x}$ & & & 20252 \\
\hline Eupatorium crenulatum Gardn. & $1,2,3$ & Arb & $\mathrm{x}$ & $\mathrm{x}$ & $\mathrm{x}$ & 17989 \\
\hline Eupatorium grandiflorum Hook. & 1,2 & Sub & $\mathrm{x}$ & & & 19582 \\
\hline Eupatorium hirsutum Gardn. & 1 & Sub & $\mathrm{x}$ & & & 22750 \\
\hline Eupatorium kleinioides H.B.K. & $2,3,4$ & Erv & $\mathrm{x}$ & $\mathrm{x}$ & & 17982 \\
\hline Eupatorium laevigatum Lam. & $1,2,3,4$ & Sub & $\mathrm{x}$ & $\mathrm{x}$ & $\mathrm{x}$ & 20744 \\
\hline Eupatorium mollicomum B.L. Robinson & 1 & Erv & $\mathrm{x}$ & & & 18602 \\
\hline Eupatorium oxyleps DC. & 1 & Sub & $\mathrm{x}$ & & & 17994 \\
\hline Eupatorium pedale Sch. Bip. ex Baker & $1,2,3$ & Sub & $\mathrm{x}$ & $\mathrm{x}$ & & 20152 \\
\hline Eupatorium purpurascens Sch. Bip. & 2,3 & Sub & $\mathrm{x}$ & $\mathrm{x}$ & & 23504 \\
\hline Eupatorium stachyophyllum Spreng. & $1,2,3,4$ & Sub & $\mathrm{x}$ & $\mathrm{x}$ & & 20319 \\
\hline Eupatorium tremulum Gardn. & 3,4 & Arb & $\mathrm{x}$ & $\mathrm{x}$ & & 19978 \\
\hline Mikania cordifolia (L.f.) Willd. & $1,2,3,4$ & Lia & $\mathrm{x}$ & $\mathrm{x}$ & $\mathrm{x}$ & 19971 \\
\hline Mikania officinalis Mart. & $1,3,4$ & Lia & $\mathrm{x}$ & $\mathrm{x}$ & & 19884 \\
\hline Mikania psilostachya DC. & 3 & Lia & & $\mathrm{x}$ & & 22648 \\
\hline Orthopappus angustifolius (Sw.) Gleason & 2 & Erv & $\mathrm{x}$ & & & 18951 \\
\hline Pterocaulon rugosum (Vahl.) Malme & 2 & Erv & $\mathrm{x}$ & & & 19491 \\
\hline Riencourtia oblongifolia Gardn. & 1 & Sub & $\mathrm{x}$ & & & 19796 \\
\hline Stenocline chionae DC. & 3 & Erv & $\mathrm{x}$ & & & 19593 \\
\hline Stevia collina Gardn. & 3,4 & Sub & $\mathrm{x}$ & $\mathrm{x}$ & & 22653 \\
\hline Stevia crenulata Baker & 3,4 & Sub & & $\mathrm{x}$ & & 22841 \\
\hline Trichogonia salviaefolia Gardn. & 2,4 & Erv & $\mathrm{x}$ & & & 22649 \\
\hline Vernonia cuneifolia Gardn. & 4 & Sub & & $\mathrm{x}$ & & 21231 \\
\hline Vernonia echitifolia Mart. ex DC. & $2,3,4$ & Sub & $\mathrm{x}$ & $\mathrm{x}$ & $\mathrm{x}$ & 22893 \\
\hline Vernonia ferruginea Less. & 4 & Arb & $\mathrm{x}$ & & & 18123 \\
\hline Vernonia fruticulosa Mart. ex DC. & 1 & Sub & $\mathrm{x}$ & & & 23588 \\
\hline Vernonia glabrata Less. & 1 & Arb & $\mathrm{x}$ & $\mathrm{x}$ & & 22195 \\
\hline Vernonia herbacea (Vell.) Rusby & 1 & Erv & $\mathrm{x}$ & & & 17229 \\
\hline Vernonia obtusata Less. & 3 & Arb & $\mathrm{x}$ & & & 22846 \\
\hline Vernonia phosphorea (Vell.) Monteiro & $1,3,4$ & Sub & $\mathrm{x}$ & & & 23439 \\
\hline Vernonia polyanthes Less. & $1,3,4$ & Sub & $\mathrm{x}$ & & & 20763 \\
\hline Vernonia psilostachya DC. & 4 & Sub & $\mathrm{x}$ & & & 22501 \\
\hline Vernonia sp.1 & 4 & Sub & $\mathrm{x}$ & $\mathrm{x}$ & & 21108 \\
\hline
\end{tabular}


Tabela 1 (cont.)

\begin{tabular}{|c|c|c|c|c|c|c|}
\hline Famílias / espécies & Veredas & Hábito & B & $\mathrm{M}$ & $\mathrm{F}$ & $\mathrm{N}$ \\
\hline \multicolumn{7}{|l|}{ ASTERACEAE } \\
\hline Vernonia sp.2 & 3,4 & Sub & $\mathrm{x}$ & $\mathrm{x}$ & & 23208 \\
\hline Vernonia viscidula Less. & 1 & Sub & & $\mathrm{x}$ & & 20921 \\
\hline Viguiera discolor Baker & 1 & Erv & $\mathrm{x}$ & & & 17231 \\
\hline Wedelia sp. & $1,3,4$ & Erv & & $\mathrm{x}$ & $\mathrm{x}$ & 22329 \\
\hline \multicolumn{7}{|l|}{ BEGONIACEAE } \\
\hline Begonia cucullata Willd. & 2,4 & Erv & & $\mathrm{x}$ & $\mathrm{x}$ & 21704 \\
\hline \multicolumn{7}{|l|}{ BIGNONIACEAE } \\
\hline Arrabidaea cf. multiflora Bureau \& K. Schum. & 4 & Lia & & & $\mathrm{x}$ & 18620 \\
\hline Tabebuia insignis (Miq.) Sandw. var. insignis & 2 & Arv & $\mathrm{x}$ & & & 18125 \\
\hline \multicolumn{7}{|l|}{ BORAGINACEAE } \\
\hline Heliotropium filiforme H.B.K. & 1 & Erv & $\mathrm{x}$ & & & 20061 \\
\hline \multicolumn{7}{|l|}{ BROMELIACEAE } \\
\hline Ananas ananassoides (Baker) L.B. Smith & 4 & Erv & $\mathrm{x}$ & & & 21292 \\
\hline \multicolumn{7}{|l|}{ BURMANNIACEAE } \\
\hline Burmannia flava Mart. & 1,4 & Erv & & $\mathrm{x}$ & & 23242 \\
\hline \multicolumn{7}{|l|}{ CAESALPINIACEAE } \\
\hline Bauhinia brevipes Vog. & 2 & Arb & $\mathrm{x}$ & & & 21015 \\
\hline Chamaecrista cathartica (Mart.) Irwin \& Barn. & 4 & Arb & $\mathrm{x}$ & & & 23492 \\
\hline Chamaecrista desvauxii (Collad.) Killip var. claussenii & 1 & Sub & & $\mathrm{x}$ & & 19595 \\
\hline Chamaecrista desvauxii (Collad.) Killip var. desvauxii & 1,4 & Sub & $\mathrm{x}$ & & & 22492 \\
\hline $\begin{array}{l}\text { Chamaecrista desvauxii (Collad.) Killip } \\
\text { var. glauca (Hassl.) Irwin \& Barn. }\end{array}$ & 1 & Sub & $\mathrm{x}$ & & & 21033 \\
\hline $\begin{array}{l}\text { Chamaecrista desvauxii (Collad.) Killip } \\
\text { var. langsdorffii (Kunth ex Vog.) Irwin \& Barn. }\end{array}$ & 1,4 & Sub & $\mathrm{x}$ & & & 22308 \\
\hline Chamaecrista flexuosa (L.) Greene & 1,3 & Sub & $\mathrm{x}$ & & & 20266 \\
\hline Chamaecrista nictitans (L.) Moench. & 3 & Erv & $\mathrm{x}$ & & & 19855 \\
\hline Chamaecrista rotundifolia (Pers.) Greene & 1 & Sub & & $\mathrm{x}$ & & 19842 \\
\hline Chamaecrista viscosa (H.B.K.) Irwin \& Barn. & 4 & Arb & $\mathrm{x}$ & & & 21114 \\
\hline Senna pendula Irwin \& Barn. & $1,2,4$ & Arb & $\mathrm{x}$ & $\mathrm{x}$ & $\mathrm{x}$ & 20928 \\
\hline \multicolumn{7}{|l|}{ CAMPANULACEAE } \\
\hline Centropogon cornutus (L.) Druce & 2 & Sub & & $\mathrm{x}$ & & 17798 \\
\hline \multicolumn{7}{|l|}{ CECROPIACEAE } \\
\hline Cecropia pachystachya Tréc. & 2,3 & Arv & & & $\mathrm{x}$ & 21013 \\
\hline \multicolumn{7}{|l|}{ CHLORANTHACEAE } \\
\hline Hedyosmum brasiliense Mart. ex Miq. & 1,3 & Arv & & & $\mathrm{x}$ & 20420 \\
\hline \multicolumn{7}{|l|}{ CLUSIACEAE } \\
\hline Clusia criuva Camb. & 1,3 & Arb & & & $\mathrm{x}$ & 18633 \\
\hline \multicolumn{7}{|l|}{ COMMELINACEAE } \\
\hline Commelina sp. & 2 & Erv & & & $\mathrm{x}$ & 21171 \\
\hline Dichorisandra hexandra (Aubl.) Standl. & $1,2,3,4$ & Erv & & $\mathrm{x}$ & & 21479 \\
\hline \multicolumn{7}{|l|}{ CONVOLVULACEAE } \\
\hline Evolvulus lagopodioides Meissn. & 1,4 & Erv & $\mathrm{x}$ & & & 22299 \\
\hline Evolvulus pterocaulon Moric. & $1,3,4$ & Sub & $\mathrm{x}$ & & & 22747 \\
\hline Evolvulus sp. & 1,4 & Erv & $\mathrm{x}$ & & & 20312 \\
\hline Ipomea procurrens Meissn. & 4 & Erv & & $\mathrm{x}$ & & 21842 \\
\hline Ipomea sp. & 2 & Lia & & & $\mathrm{x}$ & 23539 \\
\hline \multicolumn{7}{|l|}{ CUCURBITACEAE } \\
\hline Caiaponia espelina (Manso) Cogn. & 1 & Lia & $\mathrm{x}$ & & & 21786 \\
\hline \multicolumn{7}{|l|}{ CUSCUTACEAE } \\
\hline Cuscuta racemosa Mart. & 1 & Par & $\mathrm{x}$ & $\mathrm{x}$ & & 19890 \\
\hline
\end{tabular}


(cont.)

\begin{tabular}{|c|c|c|c|c|c|c|}
\hline Famílias / espécies & Veredas & Hábito & $\mathrm{B}$ & M & $\mathrm{F}$ & $\mathrm{N}$ \\
\hline \multicolumn{7}{|l|}{ CYPERACEAE } \\
\hline Ascolepis brasiliensis (Kunth) Benth. ex C.B. Clarke * & $1,2,3,4$ & Erv & $\mathrm{x}$ & $\mathrm{x}$ & & 18858 \\
\hline Bulbostylis hirtella Nees * & $1,2,4$ & Erv & $\mathrm{x}$ & $\mathrm{x}$ & & 22248 \\
\hline Bulbostylis jacobinae Lindm. & 1 & Erv & $\mathrm{x}$ & & & 17258 \\
\hline Bulbostylis junciformis C.B. Clarke * & $1,2,3,4$ & Erv & $\mathrm{x}$ & $\mathrm{x}$ & & 19571 \\
\hline Bulbostylis scabra Lindm. * & 1,4 & Erv & $\mathrm{x}$ & $\mathrm{x}$ & & 19732 \\
\hline Bulbostylis sellowiana (Kunth) Palla * & 1 & Erv & & $\mathrm{x}$ & $\mathrm{x}$ & 20903 \\
\hline Bulbostylis sphaerocephala C.B. Clarke * & 1,4 & Erv & & $\mathrm{x}$ & & 18886 \\
\hline Calyptrocarya glomerulata (Brongn.) Urban & 1,2 & Erv & & $\mathrm{x}$ & & 18847 \\
\hline Cyperus aggregatus (Willd.) Endl. & 2 & Erv & $\mathrm{x}$ & & & 19507 \\
\hline Cyperus cf. tener Ness \& Ehrenb. ex Boeck. * & $1,2,3,4$ & Erv & $\mathrm{x}$ & $\mathrm{x}$ & $\mathrm{x}$ & 17918 \\
\hline Cyperus haspan Benth. & $1,2,3,4$ & Erv & & $\mathrm{x}$ & $\mathrm{x}$ & 21284 \\
\hline Cyperus lanceolatus Poir. & 2 & Erv & & $\mathrm{x}$ & & 20685 \\
\hline Cyperus laxus Griseb. & $1,2,3,4$ & Erv & $\mathrm{x}$ & $\mathrm{x}$ & & 21863 \\
\hline Cyperus luzulae Hochst. ex Steud. & 1 & Erv & $\mathrm{x}$ & $\mathrm{x}$ & & 21524 \\
\hline Cyperus meyenianus Kunth & $1,2,3$ & Erv & $\mathrm{x}$ & $\mathrm{x}$ & $\mathrm{x}$ & 20388 \\
\hline Cyperus sp.1 & 2 & Erv & & & $\mathrm{x}$ & 21740 \\
\hline Cyperus sp.2 & 2 & Erv & & $\mathrm{x}$ & & 19664 \\
\hline Cyperus surinamensis Rottb. & 1 & Erv & & $\mathrm{x}$ & & 17897 \\
\hline Eleocharis capillacea Kunth * & $1,2,3,4$ & Erv & & $\mathrm{x}$ & & 22770 \\
\hline Eleocharis filiculmis Kunth & $2,3,4$ & Erv & $\mathrm{x}$ & $\mathrm{x}$ & $\mathrm{x}$ & 21543 \\
\hline Eleocharis geniculata $\mathrm{R} . \mathrm{Br}$. & 2,4 & Erv & $\mathrm{x}$ & $\mathrm{x}$ & $\mathrm{x}$ & 21384 \\
\hline Eleocharis obtusetrigona Steud. & 3 & Erv & & $\mathrm{x}$ & & 21754 \\
\hline Eleocharis sp.1 & 3 & Erv & & $\mathrm{x}$ & & 21389 \\
\hline Eleocharis sp. $2 *$ & 2 & Erv & & $\mathrm{x}$ & & 22769 \\
\hline Exachogyne amazonica C.B. Clarke & 1 & Erv & & $\mathrm{x}$ & & 22410 \\
\hline Fimbristylis autumnalis (L.) Roem. \& Schult. & 2,4 & Erv & $\mathrm{X}$ & $\mathrm{x}$ & & 20382 \\
\hline Fimbristylis complanata (Ritz) Link & $2,3,4$ & Erv & & $\mathrm{x}$ & & 21545 \\
\hline Fimbristylis dichotoma (L.) Vahl. & 2 & Erv & & $\mathrm{x}$ & & 20145 \\
\hline Fuirena incompleta Nees & $1,2,3,4$ & Erv & $\mathrm{x}$ & $\mathrm{x}$ & $\mathrm{x}$ & 23431 \\
\hline Kyllinga odorata H.B.K. & 2,3 & Erv & $\mathrm{x}$ & $\mathrm{x}$ & & 22909 \\
\hline Lipocarpha sellowiana Kunth * & $1,2,3,4$ & Erv & & $\mathrm{x}$ & & 20489 \\
\hline Lipocarpha sp. * & 2 & Erv & $\mathrm{x}$ & $\mathrm{x}$ & & 18898 \\
\hline Rhynchospora albiceps Kunth & $1,2,3,4$ & Erv & $\mathrm{x}$ & $\mathrm{x}$ & & 21604 \\
\hline Rhynchospora cf. emaciata Boeck. * & $1,2,3,4$ & Erv & $\mathrm{x}$ & $\mathrm{x}$ & $\mathrm{x}$ & 18867 \\
\hline Rhynchospora consanguinea (Kunth) Boeck. * & $2,3,4$ & Erv & & $\mathrm{x}$ & & 17290 \\
\hline Rhynchospora globosa (Kunth) Roem. \& Schult. * & $1,2,3,4$ & Erv & $\mathrm{x}$ & $\mathrm{x}$ & & 22381 \\
\hline Rhynchospora nervosa Boeck. & 2 & Erv & $\mathrm{x}$ & $\mathrm{x}$ & & 20320 \\
\hline Rhynchospora rigida Boeck. & 4 & Erv & & $\mathrm{x}$ & & 22698 \\
\hline Rhynchospora riparia Boeck. & 1 & Erv & & $\mathrm{x}$ & & 19568 \\
\hline Rhynchospora robusta (Kunth) Schnee & $1,2,3,4$ & Erv & & $\mathrm{x}$ & & 17294 \\
\hline Rhynchospora rugosa (Vahl.) Gale & $1,2,3,4$ & Erv & $\mathrm{x}$ & $\mathrm{x}$ & & 21094 \\
\hline Rhynchospora sp.1 & 2 & Erv & & $\mathrm{x}$ & & 21743 \\
\hline Rhynchospora sp.2 & 2 & Erv & $\mathrm{x}$ & $\mathrm{x}$ & & 22772 \\
\hline Rhynchospora sp.3 & 1 & Erv & $\mathrm{x}$ & & & 18882 \\
\hline Rhynchospora sp.4 & 4 & Erv & & $\mathrm{x}$ & & 22508 \\
\hline Rhynchospora sp.5 & 4 & Erv & & $\mathrm{x}$ & & 22695 \\
\hline Rhynchospora sp.6 & 4 & Erv & $\mathrm{x}$ & $\mathrm{x}$ & & 22508 \\
\hline Rhynchospora tenuis Link. * & $1,2,3,4$ & Erv & $\mathrm{x}$ & $\mathrm{x}$ & $\mathrm{x}$ & 17896 \\
\hline Rhynchospora velutina (Nees) Schnee & $1,2,3,4$ & Erv & $\mathrm{x}$ & $\mathrm{x}$ & $\mathrm{x}$ & 23191 \\
\hline Scleria hirtella Sw. & $1,2,3$ & Erv & $\mathrm{x}$ & $\mathrm{x}$ & $\mathrm{x}$ & 20577 \\
\hline Scleria macrophylla J. Presl \& C. Presl & 1 & Erv & $\mathrm{x}$ & $\mathrm{x}$ & $\mathrm{x}$ & 20238 \\
\hline
\end{tabular}


(cont.)

\begin{tabular}{|c|c|c|c|c|c|c|}
\hline Famílias / espécies & Veredas & Hábito & $\mathrm{B}$ & M & $\mathrm{F}$ & $\mathrm{N}$ \\
\hline \multicolumn{7}{|l|}{ CYPERACEAE } \\
\hline Scleria mitis Berg & 4 & Erv & & & \multirow[t]{3}{*}{$\mathrm{x}$} & 18861 \\
\hline Scleria scabra Willd. & 4 & Erv & $\mathrm{x}$ & & & 18873 \\
\hline Scleria sp. & 1 & Erv & & & & 18875 \\
\hline \multicolumn{7}{|l|}{ DILLENIACEAE } \\
\hline Curatella americana $\mathrm{L}$. & 4 & Arv & $\mathrm{x}$ & & & 20807 \\
\hline Davilla elliptica A. St.-Hil. & $1,2,4$ & Arb & $\mathrm{x}$ & & & 22741 \\
\hline \multicolumn{7}{|l|}{ DIOSCOREACEAE } \\
\hline Dioscorea polygonoides H \& B & 2 & Lia & & & $\mathrm{x}$ & 23525 \\
\hline \multicolumn{7}{|l|}{ DROSERACEAE } \\
\hline Drosera communis A. St.-Hil. & $1,2,3$ & Erv & & $\mathrm{x}$ & & 23033 \\
\hline \multicolumn{7}{|l|}{ EQUISETACEAE } \\
\hline Equisetum giganteum $\mathrm{L}$. & 2 & Erv & & $\mathrm{x}$ & $\mathrm{x}$ & 19879 \\
\hline \multicolumn{7}{|l|}{ ERICACEAE } \\
\hline Gaylussacia brasiliensis (Spreng.) Meissn. & 1 & Arb & & & $\mathrm{x}$ & 21185 \\
\hline Leucothoe chapadense Kinoshita-Gouvêa & 1 & Sub & & $\mathrm{x}$ & $\mathrm{x}$ & 21027 \\
\hline \multicolumn{7}{|l|}{ ERIOCAULACEAE } \\
\hline Eriocaulon elichrysoides Kunth & 1,2 & Erv & & & $\mathrm{x}$ & 21053 \\
\hline Eriocaulon modestum Kunth & $1,2,3$ & Erv & & $\mathrm{x}$ & $\mathrm{x}$ & 20913 \\
\hline Eriocaulon $\mathrm{sp} .1$ & 2 & Erv & & & $\mathrm{x}$ & 20888 \\
\hline Eriocaulon sp. 2 & 1 & Erv & & & $\mathrm{x}$ & 20905 \\
\hline Eriocaulon sp. 3 & 1 & Erv & & $\mathrm{x}$ & & 19598 \\
\hline Paepalanthus aquatile (Koern.) Ruhl. & 1 & Erv & & & $\mathrm{x}$ & 20914 \\
\hline Paepalanthus cachambuensis Alv. Silv. & 1 & Erv & & $\mathrm{x}$ & & 21115 \\
\hline Paepalanthus flaccidus (Bong.) Kunth & $1,2,3,4$ & Erv & & $\mathrm{x}$ & $\mathrm{x}$ & 19938 \\
\hline Paepalanthus geniculatus Kunth & $1,2,3,4$ & Erv & & $\mathrm{x}$ & $\mathrm{x}$ & 17076 \\
\hline Paepalanthus scholiophyllus Ruhl. & 1 & Erv & $\mathrm{x}$ & & & 16963 \\
\hline Paepalanthus speciosus (Bong.) Koern. & 2 & Erv & & $\mathrm{x}$ & & 19499 \\
\hline Syngonanthus appressus (Koern.) Ruhl. & 1 & Erv & $\mathrm{x}$ & $\mathrm{x}$ & & 21214 \\
\hline Syngonanthus caulescens (Poir.) Ruhl. & $1,2,3,4$ & Erv & $\mathrm{x}$ & $\mathrm{x}$ & $\mathrm{x}$ & 20659 \\
\hline Syngonanthus densiflorus (Koern.) Ruhl. & $1,2,3,4$ & Erv & $\mathrm{x}$ & $\mathrm{x}$ & & 19737 \\
\hline Syngonanthus fuscescens Ruhl. & 2,3 & Erv & & $\mathrm{x}$ & $\mathrm{x}$ & 20243 \\
\hline Syngonanthus gracilis Ruhl. var. aurea Ruhl. & 1 & Erv & & & $\mathrm{x}$ & 20909 \\
\hline Syngonanthus nitens (Bong.) Ruhl. & $2,3,4$ & Erv & & $\mathrm{x}$ & $\mathrm{x}$ & 20550 \\
\hline Syngonanthus sp. & 1,3 & Erv & & $\mathrm{x}$ & & 20904 \\
\hline Syngonanthus widgrenianus (Koern.) Ruhl. & 1 & Erv & & & $\mathrm{x}$ & 21055 \\
\hline Syngonanthus xeranthemoides (Bong.) Ruhl. & 2 & Erv & $\mathrm{x}$ & $\mathrm{x}$ & $\mathrm{x}$ & 19736 \\
\hline \multicolumn{7}{|l|}{ ERYTHROXYLACEAE } \\
\hline Erythroxylum campestris A. St.-Hil. & 3 & Arb & $\mathrm{x}$ & & & 17277 \\
\hline \multicolumn{7}{|l|}{ EUPHORBIACEAE } \\
\hline Acalypha communis M. Arg. & 2 & Sub & $\mathrm{x}$ & & & 17810 \\
\hline Acalypha sp. & 2 & Sub & $\mathrm{x}$ & & & 19880 \\
\hline Chamaesyce potentilloides (Boiss.) Croizat & $1,2,3,4$ & Erv & $\mathrm{x}$ & & & 21007 \\
\hline Croton lundianus (F. Didr.) M. Arg. & $1,2,4$ & Sub & $\mathrm{x}$ & $\mathrm{x}$ & & 22332 \\
\hline Croton sclerocalyx (F. Didr.) M. Arg. & 3 & Erv & $\mathrm{x}$ & $\mathrm{x}$ & & 21894 \\
\hline Euphorbia coecorum M. Arg. & $1,2,3$ & Erv & $\mathrm{x}$ & $\mathrm{x}$ & & 21415 \\
\hline Phyllanthus orbiculatus L.C. Rich. & $1,3,4$ & Sub & $\mathrm{x}$ & & & 20091 \\
\hline Phyllanthus perpusillus Baill. & 1,3 & Sub & $\mathrm{x}$ & $\mathrm{x}$ & & 18651 \\
\hline Sapium glandulatum (Vell.) Pax & 2 & Arb & $\mathrm{x}$ & $\mathrm{x}$ & $\mathrm{x}$ & 20417 \\
\hline Sebastiania cf. klotzschiana M. Arg. & 2 & Sub & & & $\mathrm{x}$ & 18149 \\
\hline Sebastiania myrtilloides (Mart.) Pax & $1,3,4$ & Sub & $\mathrm{x}$ & & & 22305 \\
\hline
\end{tabular}


(cont.)

\begin{tabular}{|c|c|c|c|c|c|c|}
\hline Famílias / espécies & Veredas & Hábito & $\mathrm{B}$ & M & $\mathrm{F}$ & $\mathrm{N}$ \\
\hline \multicolumn{7}{|l|}{ FABACEAE } \\
\hline Aeschynomene falcata (Poir.) DC. & 2 & Erv & & & \multirow[t]{11}{*}{$\mathrm{x}$} & 22344 \\
\hline Aeschynomene paniculata Willd. ex Vog. & $1,3,4$ & Sub & $\mathrm{x}$ & $\mathrm{x}$ & & 20025 \\
\hline Camptosema coriaceum ( Nees \& Mart.) Benth. & 1 & Arb & $\mathrm{x}$ & & & 20089 \\
\hline Centrosema pascuorum Mart. & 4 & Sub & $\mathrm{x}$ & & & 19604 \\
\hline Clitoria guianensis (Aubl.) Benth. & 1,4 & Sub & $\mathrm{x}$ & & & 17235 \\
\hline Collaea speciosa DC. & 2 & Arb & $\mathrm{x}$ & & & 18152 \\
\hline Crotalaria brachystachya Benth. & $1,3,4$ & Arb & $\mathrm{x}$ & $\mathrm{x}$ & & 22491 \\
\hline Crotalaria pilosa Miller & 3 & Sub & $\mathrm{x}$ & & & 19866 \\
\hline Desmodium barbatum (L.) Benth. & $2,3,4$ & Sub & $\mathrm{x}$ & & & 19602 \\
\hline Desmodium discolor Vog. & 4 & Erv & $\mathrm{x}$ & & & 19605 \\
\hline Desmodium incanum (Sw.) DC. & 2 & Sub & $\mathrm{x}$ & & & 18661 \\
\hline Desmodium platycarpum Benth. & 2,4 & Erv & $\mathrm{x}$ & $\mathrm{x}$ & \multirow[t]{12}{*}{$\mathrm{x}$} & 19741 \\
\hline Eriosema benthamianum Mart. ex Benth. & 3 & Sub & $\mathrm{x}$ & & & 20812 \\
\hline Eriosema sp. & 1,3 & Sub & $\mathrm{x}$ & $\mathrm{x}$ & & 20811 \\
\hline Indigofera bongardiana (Kuntze) Burkart & 1 & Sub & $\mathrm{x}$ & & & 16727 \\
\hline Stylosanthes acuminata M.B. Ferr. \& S. Costa & 1 & Erv & $\mathrm{x}$ & & & 22739 \\
\hline Stylosanthes gracilis H.B.K. & $1,3,4$ & Sub & $\mathrm{x}$ & & & 21536 \\
\hline Stylosanthes guianensis (Aubl.) Sw. & $1,2,3,4$ & Sub & $\mathrm{x}$ & & & 20331 \\
\hline Stylosanthes nunoi Brandão & 2 & Sub & $\mathrm{x}$ & & & 19740 \\
\hline Stylosanthes sp. & 2 & Sub & $\mathrm{x}$ & & & 21583 \\
\hline Tephrosia adunca Benth. & 3 & Erv & $\mathrm{x}$ & & & 21082 \\
\hline Zornia latifolia Sm. & 1,4 & Erv & $\mathrm{x}$ & $\mathrm{x}$ & & 22428 \\
\hline Zornia reticulata $\mathrm{Sm}$. & 1 & Erv & $\mathrm{x}$ & & & 19847 \\
\hline \multicolumn{7}{|l|}{ GENTIANACEAE } \\
\hline Curtia tenuifolia (Aubl.) Knobl. & 1 & Erv & & $\mathrm{x}$ & \multirow{8}{*}{$\mathrm{x}$} & 21048 \\
\hline Irlbachia coaerulescens (Aubl.) Griseb. & $1,2,3,4$ & Sub & $\mathrm{x}$ & $\mathrm{x}$ & & 23045 \\
\hline Irlbachia alata (Aubl.) Maas & 1 & Erv & $\mathrm{x}$ & $\mathrm{x}$ & & 19813 \\
\hline Irlbachia oblongifolia (Mart.) Maas & 1 & Erv & $\mathrm{x}$ & & & 18263 \\
\hline Lisianthius sp. & 1 & Erv & $\mathrm{x}$ & & & 18030 \\
\hline Schultesia heterophylla Miq. & $2,3,4$ & Erv & $\mathrm{x}$ & $\mathrm{x}$ & & 19669 \\
\hline Schultesia aptera Cham. & $1,2,3,4$ & Erv & & $\mathrm{x}$ & & 19764 \\
\hline Schultesia gracilis Mart. & 2,4 & Erv & $\mathrm{x}$ & $\mathrm{x}$ & & 20611 \\
\hline \multicolumn{7}{|l|}{ GESNERIACEAE } \\
\hline Sinningia elatior (Kunth) Chautems & $1,3,4$ & Erv & $\mathrm{x}$ & $\mathrm{x}$ & $\mathrm{X}$ & 21722 \\
\hline \multicolumn{7}{|l|}{ GLEICHENIACEAE } \\
\hline Dicranopteris flexuosa (Schrad.) Underw. & $1,2,3,4$ & Erv & $\mathrm{x}$ & $\mathrm{x}$ & $\mathrm{x}$ & 20633 \\
\hline \multicolumn{7}{|l|}{ HELICONIACEAE } \\
\hline Heliconia psittacorum Sessé \& Moc. & 2 & Erv & & & $\mathrm{x}$ & 18326 \\
\hline \multicolumn{7}{|l|}{ HYDROCARITACEAE } \\
\hline Beneditaea brasiliensis (Planch.) Toledo & 2 & Erv & & & $\mathrm{x}$ & 23020 \\
\hline \multicolumn{7}{|l|}{ HYDROPHYLLACEAE } \\
\hline Hydrolea cf. spinosa L. & 2 & Erv & & & $\mathrm{x}$ & 21173 \\
\hline \multicolumn{7}{|l|}{ IRIDACEAE } \\
\hline Cipura paludosa Aubl. & $1,2,3$ & Erv & $\mathrm{x}$ & $\mathrm{x}$ & & 21179 \\
\hline Sisyrinchium incurvatum Gard. & $1,2,3,4$ & Erv & $\mathrm{x}$ & $\mathrm{x}$ & & 22936 \\
\hline Sisyrinchium luzula Klotz. ex Klatt & $1,2,3$ & Erv & $\mathrm{x}$ & $\mathrm{x}$ & & 21427 \\
\hline Sisyrinchium vaginatum Spreng. & 1,3 & Erv & $\mathrm{x}$ & $\mathrm{x}$ & & 20293 \\
\hline Trimezia juncifolia (Klatt) Benth. \& Hook. f. & 1,2 & Erv & & $\mathrm{x}$ & & 21413 \\
\hline Trimezia sp. & 1 & Erv & $\mathrm{x}$ & $\mathrm{x}$ & & 18681 \\
\hline \multicolumn{7}{|l|}{ LAMIACEAE } \\
\hline Eriope crassipes Benth. & 1 & Sub & $\mathrm{x}$ & & & 16976 \\
\hline
\end{tabular}


(cont.)

\begin{tabular}{|c|c|c|c|c|c|c|}
\hline Famílias / espécies & Veredas & Hábito & B & $\mathrm{M}$ & $\mathrm{F}$ & $\mathrm{N}$ \\
\hline \multicolumn{7}{|l|}{ LAMIACEAE } \\
\hline Hyptis althaeaefolia Pohl ex Benth. & 2 & Sub & $\mathrm{x}$ & $\mathrm{x}$ & & 20217 \\
\hline Hyptis carpinifolia Benth. & 2 & Arb & $\mathrm{x}$ & $\mathrm{x}$ & & 20227 \\
\hline Hyptis cf. interrupta Pohl ex Benth. & 1,2 & Erv & $\mathrm{x}$ & $\mathrm{x}$ & & 22792 \\
\hline Hyptis cf. tenuifolia Epling & 2 & Sub & $\mathrm{x}$ & $\mathrm{x}$ & & 19672 \\
\hline Hyptis crenata Pohl ex Benth. & $2,3,4$ & Sub & $\mathrm{x}$ & $\mathrm{x}$ & & 22823 \\
\hline Hyptis lantanaefolia Poit. & $1,2,3,4$ & Sub & $\mathrm{x}$ & $\mathrm{x}$ & & 22624 \\
\hline Hyptis linarioides Pohl ex Benth. & $1,3,4$ & Sub & $\mathrm{x}$ & & & 22288 \\
\hline Hyptis lippioides Pohl ex Benth. & 2 & Sub & $\mathrm{x}$ & $\mathrm{x}$ & & 22971 \\
\hline Hyptis nudicaulis Benth. & 4 & Erv & & $\mathrm{x}$ & & 23235 \\
\hline Hyptis paludosa A. St.-Hil. ex Benth. & $1,2,4$ & Sub & $\mathrm{x}$ & $\mathrm{x}$ & $\mathrm{x}$ & 22234 \\
\hline Hyptis sinuata Pohl ex Benth. & 2 & Sub & & $\mathrm{x}$ & & 22641 \\
\hline Hyptis subrotunda Pohl ex Benth. & $1,2,3,4$ & Sub & $\mathrm{x}$ & $\mathrm{x}$ & & 23491 \\
\hline Hyptis tenuifolia Epling & 2 & Sub & & $\mathrm{x}$ & & 19672 \\
\hline Hyptis velutina Pohl & 1 & Sub & $\mathrm{x}$ & $\mathrm{x}$ & $\mathrm{x}$ & 20047 \\
\hline Hyptis villosa Pohl ex Benth. & 2 & Sub & $\mathrm{x}$ & & & 22972 \\
\hline Hyptis virgata Benth. & 1 & Sub & $\mathrm{x}$ & & & 17230 \\
\hline Peltodon tomentosus Pohl & 1 & Erv & $\mathrm{x}$ & & & 22593 \\
\hline Salvia scabrida Pohl & 4 & Sub & & $\mathrm{x}$ & $\mathrm{x}$ & 22822 \\
\hline \multicolumn{7}{|l|}{ LAURACEAE } \\
\hline Ocotea corymbosa (Miers) Mez & 2 & Arv & $\mathrm{x}$ & & & 22994 \\
\hline \multicolumn{7}{|l|}{ LENTIBULARIACEAE } \\
\hline Utricularia bicolor A. St.-Hil. \& Girard & $1,2,3,4$ & Erv & $\mathrm{x}$ & $\mathrm{x}$ & & 23465 \\
\hline Utricularia nana A. St.-Hil. \& Girard & $1,2,3,4$ & Erv & $\mathrm{x}$ & $\mathrm{x}$ & $\mathrm{x}$ & 22776 \\
\hline Utricularia purpureocaerulea A. St.-Hil. & $1,2,3,4$ & Erv & & $\mathrm{x}$ & $\mathrm{x}$ & 17302 \\
\hline \multicolumn{7}{|l|}{ LILIACEAE } \\
\hline Alstroemeria sp. & 4 & Erv & & $\mathrm{x}$ & & 21465 \\
\hline Liliaceae sp. & $1,3,4$ & Erv & & $\mathrm{x}$ & $\mathrm{x}$ & 21815 \\
\hline \multicolumn{7}{|l|}{ LORANTHACEAE } \\
\hline Phoradendron mucronatum (DC.) Krug. \& Urban & 2 & Par & & $\mathrm{x}$ & & 21417 \\
\hline \multicolumn{7}{|l|}{ LYCOPODIACEAE } \\
\hline Lycopodiella alopecuroides (L.) Cranfill & 1,4 & Erv & $\mathrm{x}$ & $\mathrm{x}$ & $\mathrm{x}$ & 22888 \\
\hline Lycopodiella camporum B. Øllg. \& P.G. Wind. & $1,2,3,4$ & Erv & $\mathrm{x}$ & $\mathrm{x}$ & & 18705 \\
\hline Lycopodiella cernua (L.) Pichi-Sermolli & 4 & Erv & & $\mathrm{x}$ & & 18397 \\
\hline Lycopodium carolinianum $\mathrm{L}$. & $1,2,4$ & Erv & $\mathrm{x}$ & $\mathrm{x}$ & & 23231 \\
\hline \multicolumn{7}{|l|}{ LYTHRACEAE } \\
\hline Cuphea cf. grandiflora Pohl ex Koehne & 2 & Sub & $\mathrm{x}$ & $\mathrm{x}$ & $\mathrm{x}$ & 19873 \\
\hline Cuphea $\mathrm{cf}$. sessilifolia Mart. & 3,4 & Sub & $\mathrm{x}$ & $\mathrm{x}$ & $\mathrm{x}$ & 21841 \\
\hline Cuphea linarioides Cham. \& Schlecht. & $1,2,3,4$ & Erv & $\mathrm{x}$ & $\mathrm{x}$ & & 18160 \\
\hline Diplusodon virgatus Pohl & 2,3 & Arb & $\mathrm{x}$ & $\mathrm{x}$ & & 19946 \\
\hline \multicolumn{7}{|l|}{ MALPIGHIACEAE } \\
\hline Banisteriopsis campestris (A. Juss.) Little & 1,2 & Lia & $\mathrm{x}$ & & & 21759 \\
\hline Banisteriopsis pubipetala (A. Juss.) Cuatrec. & 4 & Lia & $\mathrm{x}$ & & & 20820 \\
\hline Banisteriopsis sp. & 4 & Lia & $\mathrm{x}$ & & & 23214 \\
\hline Banisteriopsis stellaris (Griseb.) B. Gates & 1,4 & Arb & $\mathrm{x}$ & & & 20086 \\
\hline Byrsonima crassa Nied. & 3,4 & Arv & $\mathrm{x}$ & & & 23494 \\
\hline Byrsonima intermedia A. Juss. & $1,2,3,4$ & Arb & $\mathrm{x}$ & & & 21174 \\
\hline Byrsonima sp. & 1 & Sub & $\mathrm{x}$ & & & 23363 \\
\hline Camarea affinis A. St.-Hil. & 3,4 & Ver & $\mathrm{x}$ & $\mathrm{x}$ & & 22291 \\
\hline Heteropterys anoptera A. Juss. & 1,2 & Lia & $\mathrm{x}$ & & & 21195 \\
\hline Pterandra pyroidea A. Juss. & 2,4 & Arb & $\mathrm{x}$ & & & 21374 \\
\hline
\end{tabular}


(cont.)

\begin{tabular}{|c|c|c|c|c|c|c|}
\hline Famílias / espécies & Veredas & Hábito & $\mathrm{B}$ & M & $\mathrm{F}$ & $\mathrm{N}$ \\
\hline \multicolumn{7}{|l|}{ MALVACEAE } \\
\hline Pavonia rosa-campestris A. Juss. & 3,4 & Sub & $\mathrm{x}$ & & & 18712 \\
\hline Pavonia sp. & 1 & Sub & $\mathrm{x}$ & & & 22729 \\
\hline Peltaea acutifolia (Guerke) Krapov. \& Crist. & 2,4 & Arb & $\mathrm{x}$ & $\mathrm{x}$ & & 22700 \\
\hline Sida linifolia Cav. & 2,4 & Sub & $\mathrm{x}$ & $\mathrm{x}$ & & 19749 \\
\hline Sida rhombifolia L. & 4 & Sub & $\mathrm{x}$ & & & 20585 \\
\hline Sida tuberculata R.E. Fries & 4 & Sub & $\mathrm{x}$ & $\mathrm{x}$ & & 20469 \\
\hline \multicolumn{7}{|l|}{ MAYACACEAE } \\
\hline Mayaca sellowiana Kunth & $1,2,4$ & Ver & & $\mathrm{x}$ & $\mathrm{x}$ & 20128 \\
\hline \multicolumn{7}{|l|}{ MELASTOMATACEAE } \\
\hline Acisanthera alsinaefolia (Mart. \& Schr. ex DC.) Triana & $2,3,4$ & Sub & $\mathrm{x}$ & $\mathrm{x}$ & & 17849 \\
\hline Cambessedesia hilariana (Kunth) DC. & 1,2 & Ver & $\mathrm{x}$ & & & 19804 \\
\hline Desmocelis villosa (Aubl.) Naudin & $1,2,3,4$ & Sub & $\mathrm{x}$ & $\mathrm{x}$ & & 19982 \\
\hline Leandra sp. & 1 & Arb & & & $\mathrm{x}$ & 20057 \\
\hline Macairea radula (Bonpl.) DC. & $1,2,3,4$ & Sub & $\mathrm{x}$ & $\mathrm{x}$ & & 18081 \\
\hline Miconia albicans (Sw.) Triana & 2,4 & Arb & $\mathrm{x}$ & & & 21291 \\
\hline Miconia chamissois Naudin & $1,2,3,4$ & Arb & $\mathrm{x}$ & $\mathrm{x}$ & $\mathrm{x}$ & 21078 \\
\hline Miconia fallax DC. & 1,4 & Arb & $\mathrm{x}$ & & & 21096 \\
\hline Miconia rubiginosa (Bonpl.) DC & 1 & Arb & $\mathrm{x}$ & & & 20048 \\
\hline Miconia stenostachya DC. & 4 & Arb & $\mathrm{x}$ & & & 17014 \\
\hline Miconia theaezans Cogn. & $1,2,3,4$ & Arv & $\mathrm{x}$ & $\mathrm{x}$ & $\mathrm{x}$ & 22665 \\
\hline Microlicia doryphylla Naudin & $1,2,3$ & Sub & $\mathrm{x}$ & & & 18745 \\
\hline Microlicia euphorbioides Mart. & $1,2,3,4$ & Sub & $\mathrm{x}$ & & & 18717 \\
\hline Microlicia fasciculata Mart. ex Naudin & $1,2,3,4$ & Sub & $\mathrm{x}$ & & & 23425 \\
\hline Microlicia helvola (Spreng.) Triana & $1,2,3,4$ & Sub & $\mathrm{x}$ & $\mathrm{x}$ & & 22730 \\
\hline Microlicia polystemma Naudin & 1,2 & Sub & $\mathrm{x}$ & $\mathrm{x}$ & & 22618 \\
\hline Pterolepsis glomerata (Rottb.) Miq. & 2 & Sub & & $\mathrm{x}$ & $\mathrm{x}$ & 22799 \\
\hline Rhynchanthera dichotoma DC. & $2,3,4$ & Sub & $\mathrm{x}$ & $\mathrm{x}$ & $\mathrm{x}$ & 19371 \\
\hline Rhynchanthera grandiflora (Aubl.) DC. & $1,2,3,4$ & Sub & $\mathrm{x}$ & $\mathrm{x}$ & & 18746 \\
\hline Siphanthera cordata Pohl & $1,2,3,4$ & Ver & $\mathrm{x}$ & $\mathrm{x}$ & & 21107 \\
\hline Siphanthera foliosa (Naudin) Wurdack & 3,4 & Ver & $\mathrm{x}$ & & & 17034 \\
\hline Siphanthera gracillima (Naudin) Wurdack & 1 & Ver & $\mathrm{x}$ & & & 19494 \\
\hline Tibouchina candolleana (DC.) Cogn. & 1 & Arv & & $\mathrm{x}$ & & 21196 \\
\hline Tibouchina gracilis (Bonpl.) Cogn. & $1,2,3,4$ & Arb & $\mathrm{x}$ & $\mathrm{x}$ & & 22984 \\
\hline Tibouchina herbacea (DC.) Cogn. & 4 & Ver & & $\mathrm{x}$ & & 19380 \\
\hline Trembleya parviflora (D. Don.) Cogn. & 4 & Sub & $\mathrm{x}$ & & & 23478 \\
\hline Trembleya phlogiformis Mart. \& Schr. ex DC. & $1,2,3,4$ & Arb & $\mathrm{x}$ & $\mathrm{x}$ & & 21263 \\
\hline \multicolumn{7}{|l|}{ MELIACEAE } \\
\hline Guarea macrophylla Vahl & 1 & Arv & & & $\mathrm{x}$ & 20078 \\
\hline \multicolumn{7}{|l|}{ MIMOSACEAE } \\
\hline Mimosa distans Benth. & 3 & Sub & $\mathrm{x}$ & & & 22449 \\
\hline Mimosa gracilis Benth. & $1,3,4$ & Sub & $\mathrm{x}$ & & & 19827 \\
\hline $\begin{array}{l}\text { Mimosa nuda Benth. var. glaberrima } \\
\text { (Chod. \& Hassl.) Barn. }\end{array}$ & 4 & Sub & $\mathrm{x}$ & & & 21843 \\
\hline Mimosa sensitiva Lodd. & 1,4 & Sub & $\mathrm{x}$ & & & 22490 \\
\hline Mimosa setosa Benth. & $1,2,4$ & Sub & $\mathrm{x}$ & $\mathrm{x}$ & & 19863 \\
\hline \multicolumn{7}{|l|}{ MONIMIACEAE } \\
\hline Siparuna minutiflora Perkins & 3 & Arv & & $\mathrm{x}$ & & 21175 \\
\hline \multicolumn{7}{|l|}{ MYRSINACEAE } \\
\hline Myrsine cf. ferruginea (Sw.) R. Br. ex Roem. \& Schult. & 3 & Arv & & & $\mathrm{x}$ & 20214 \\
\hline Myrsine umbelata Mart. & 2 & Arv & & & $\mathrm{x}$ & 20938 \\
\hline
\end{tabular}


(cont.)

\begin{tabular}{|c|c|c|c|c|c|c|}
\hline Famílias / espécies & Veredas & Hábito & B & $\mathrm{M}$ & $\mathrm{F}$ & $\mathrm{N}$ \\
\hline \multicolumn{7}{|l|}{ MYRTACEAE } \\
\hline Blepharocalyx salicifolius (H.B.K.) O. Berg & 2 & Arv & $\mathrm{x}$ & & & 18182 \\
\hline Campomanesia pubescens (DC.) O. Berg & 2 & Arb & $\mathrm{x}$ & & & 21376 \\
\hline Eugenia calycina Cambess. & 4 & Sub & $\mathrm{x}$ & & & 21276 \\
\hline Eugenia sp. & 1,4 & Sub & $\mathrm{x}$ & & & 17246 \\
\hline Myrcia guianensis DC. & 2 & Arb & $\mathrm{x}$ & & & 18181 \\
\hline Myrcia rostrata DC. & 2 & Arv & $\mathrm{x}$ & & & 21410 \\
\hline Myrcia tomentosa (Aubl.) DC. & 2 & Arv & $\mathrm{x}$ & & & 21345 \\
\hline Myrcia variabilis Mart. ex DC. & 2,4 & Arb & $\mathrm{x}$ & & & 21375 \\
\hline Myrcia vestita DC. & 2 & Arb & $\mathrm{x}$ & & & 21404 \\
\hline Psidium guianense $\mathrm{Sw}$. & 2 & Arb & $\mathrm{x}$ & & & 21394 \\
\hline \multicolumn{7}{|l|}{ NYCTAGINACEAE } \\
\hline Neea theifera Oerst. & 2 & Arv & $\mathrm{x}$ & & & 21373 \\
\hline \multicolumn{7}{|l|}{ OCHNACEAE } \\
\hline Ouratea sp. & 1 & Arb & & & $\mathrm{x}$ & 20209 \\
\hline Sauvagesia erecta L. & $1,2,3,4$ & Sub & $\mathrm{x}$ & $\mathrm{x}$ & & 22626 \\
\hline Sauvagesia linearefolia A. St.-Hil. & 1 & Sub & $\mathrm{x}$ & $\mathrm{x}$ & & 19910 \\
\hline Sauvagesia racemosa A. St.-Hil. & $1,2,3,4$ & Sub & $\mathrm{x}$ & $\mathrm{x}$ & & 21762 \\
\hline \multicolumn{7}{|l|}{ ONAGRACEAE } \\
\hline Ludwigia filiformis (Micheli) Ramamoorthy & 2,4 & Sub & & $\mathrm{x}$ & $\mathrm{x}$ & 19874 \\
\hline Ludwigia goiazensis Ramamoorthy & 2 & Sub & & & $\mathrm{x}$ & 18308 \\
\hline Ludwigia longifolia (DC.) Hara & 1 & Sub & & $\mathrm{x}$ & & 17775 \\
\hline Ludwigia nervosa (Poir.) Hara & $1,2,3,4$ & Sub & $\mathrm{x}$ & $\mathrm{x}$ & & 20700 \\
\hline Ludwigia peruviana (L.) Hara & $1,2,3,4$ & Arb & & $\mathrm{x}$ & $\mathrm{x}$ & 22829 \\
\hline \multicolumn{7}{|l|}{ ORCHIDACEAE } \\
\hline Bletia catenulata Ruiz \& Pav. & 2 & Erv & & $\mathrm{x}$ & & 17800 \\
\hline Cyanaeorchis arundinae (Rchb.f.) Barb. Rodr. & 2,4 & Erv & & $\mathrm{x}$ & & 18370 \\
\hline Cyrtopodium hatschbachii Pabst & $1,2,3$ & Erv & & $\mathrm{x}$ & & 24405 \\
\hline Cyrtopodium paludicolum Hoehne & 3,4 & Erv & & & $\mathrm{x}$ & 21703 \\
\hline Epistephium laxiflorum Barb. Rodr. & 2 & Erv & & $\mathrm{x}$ & & 19677 \\
\hline Habenaria cf. secundiflora Barb.Rodr. & 1,4 & Erv & $\mathrm{x}$ & $\mathrm{x}$ & & 19405 \\
\hline Habenaria glazioviana Kraenzl. ex Cogn. & 3 & Erv & & $\mathrm{x}$ & & 19951 \\
\hline Habenaria nuda Lindl. & $2,3,4$ & Erv & $\mathrm{x}$ & $\mathrm{x}$ & & 20573 \\
\hline Habenaria sp. & 1 & Erv & $\mathrm{x}$ & & & 19901 \\
\hline \multicolumn{7}{|l|}{ OXALIDACEAE } \\
\hline Oxalis densiflora Sond. & $2,3,4$ & Sub & $\mathrm{x}$ & & & 19953 \\
\hline \multicolumn{7}{|l|}{ PASSIFLORACEAE } \\
\hline Passiflora gardneri Mart. & 2,4 & Lia & & $\mathrm{x}$ & $\mathrm{x}$ & 22791 \\
\hline \multicolumn{7}{|l|}{ PHYTOLACCACEAE } \\
\hline Phytolacca thryrsiflora Frenz. ex Schm. & 1 & Sub & & $\mathrm{x}$ & & 21785 \\
\hline \multicolumn{7}{|l|}{ PIPERACEAE } \\
\hline Piper corintoanum Yunck. \& Derck & $1,2,3,4$ & Arb & & $\mathrm{x}$ & $\mathrm{x}$ & 23495 \\
\hline Piper macedoi Yunck. & 2 & Erv & $\mathrm{x}$ & & $\mathrm{x}$ & 17796 \\
\hline Piper regnellii (Miq.) C. DC. & 2 & Sub & & & $\mathrm{x}$ & 18185 \\
\hline \multicolumn{7}{|l|}{ POACEAE } \\
\hline Andropogon bicornis Benth. & $1,2,3,4$ & Erv & $\mathrm{x}$ & $\mathrm{x}$ & & 19537 \\
\hline Andropogon lateralis Nees & $1,2,3,4$ & Erv & $\mathrm{x}$ & $\mathrm{x}$ & & 19480 \\
\hline Andropogon leucostachyus (Hack.) Hack. & $1,2,3,4$ & Erv & $\mathrm{x}$ & $\mathrm{x}$ & & 23335 \\
\hline Andropogon macrothrix Foum. & $1,2,3,4$ & Erv & $\mathrm{x}$ & $\mathrm{x}$ & & 19070 \\
\hline Andropogon marginatus Steud. & 3 & Erv & $\mathrm{x}$ & & & 22417 \\
\hline Andropogon selloanus Hack. & $1,2,3,4$ & Erv & $\mathrm{x}$ & $\mathrm{x}$ & & 21608 \\
\hline Andropogon sp. & 2,3 & Erv & $\mathrm{x}$ & $\mathrm{x}$ & & 20836 \\
\hline
\end{tabular}


(cont.)

\begin{tabular}{|c|c|c|c|c|c|c|}
\hline Famílias / espécies & Veredas & Hábito & B & M & $\mathrm{F}$ & $\mathrm{N}$ \\
\hline \multicolumn{7}{|l|}{ POACEAE } \\
\hline Andropogon ternatus Nees & $2,3,4$ & Erv & $\mathrm{x}$ & $\mathrm{x}$ & & 22950 \\
\hline Andropogon virgatus Desv. & $1,2,3,4$ & Erv & $\mathrm{x}$ & $\mathrm{x}$ & & 22957 \\
\hline Anthaenantiopsis trachystachya $\mathrm{Mez} *$ & $1,3,4$ & Erv & $\mathrm{x}$ & $\mathrm{x}$ & $\mathrm{x}$ & 21674 \\
\hline Aristida riparia Trin. & $1,3,4$ & Erv & $\mathrm{x}$ & $\mathrm{x}$ & & 19775 \\
\hline Aristida setifolia H.B.K. & 2 & Erv & $\mathrm{x}$ & & & 20771 \\
\hline Aristida sp. & 1 & Erv & $\mathrm{x}$ & & & 23418 \\
\hline Arthropogon filifolius Filgueiras * & 1,3 & Erv & $\mathrm{x}$ & $\mathrm{x}$ & & 21047 \\
\hline Arundinella hispida Hack. & 3 & Erv & $\mathrm{x}$ & & & 18973 \\
\hline Axonopus aureus Beauv. & 1 & Erv & $\mathrm{x}$ & $\mathrm{x}$ & & 23026 \\
\hline Axonopus brasiliensis Kuhlm. * & 1,3 & Erv & $\mathrm{x}$ & $\mathrm{x}$ & & 21876 \\
\hline Axonopus chrysoblepharis Chase * & $1,2,3,4$ & Erv & $\mathrm{x}$ & & & 20911 \\
\hline Axonopus fissifolius (Raddi) Chase & 1 & Erv & $\mathrm{x}$ & & & 18969 \\
\hline Axonopus marginatus Chase ex Hitchcock & $1,2,3$ & Erv & $\mathrm{x}$ & $\mathrm{x}$ & & 17963 \\
\hline Axonopus siccus Kuhlm. * & 1,3 & Erv & $\mathrm{x}$ & $\mathrm{x}$ & & 22609 \\
\hline Axonopus sp. & 1,4 & Erv & $\mathrm{x}$ & $\mathrm{x}$ & & 20890 \\
\hline Brachiaria decumbens Stapf. & 1 & Erv & $\mathrm{x}$ & & & 22413 \\
\hline Ctenium brevispicatum J.G. Sm. * & 1 & Erv & $\mathrm{x}$ & $\mathrm{x}$ & & 21361 \\
\hline Echinolaena inflexa Chase & $1,2,3,4$ & Erv & $\mathrm{x}$ & & & 24457 \\
\hline Elionurus adustus Ekman * & 1 & Erv & $\mathrm{x}$ & $\mathrm{x}$ & $\mathrm{x}$ & 21806 \\
\hline Elionurus muticus Kuntze * & 1,2 & Erv & $\mathrm{x}$ & $\mathrm{x}$ & & 21365 \\
\hline Eragrostis solida Nees & 1,2 & Erv & $\mathrm{x}$ & & & 20143 \\
\hline Erianthus asper Nees & 1,2 & Erv & & $\mathrm{x}$ & & 20802 \\
\hline Eriochrysis cayanensis Beauv. & $1,2,3,4$ & Erv & $\mathrm{x}$ & $\mathrm{x}$ & $\mathrm{x}$ & 18946 \\
\hline Eriochrysis laxa Swallen & 2,3 & Erv & & $\mathrm{x}$ & & 23515 \\
\hline Eriochrysis warmingiana (Hack.) Kuhlm. & $1,2,4$ & Erv & $\mathrm{x}$ & $\mathrm{x}$ & $\mathrm{x}$ & 22574 \\
\hline Hyparrhenia bracteata Stapf & $1,2,3,4$ & Erv & $\mathrm{x}$ & $\mathrm{x}$ & & 23025 \\
\hline Ichnanthus procurrens (Nees) Swallen & $1,2,3,4$ & Erv & $\mathrm{x}$ & $\mathrm{x}$ & & 21767 \\
\hline Leptocoryphium lanatum Nees * & $1,2,3,4$ & Erv & $\mathrm{x}$ & $\mathrm{x}$ & & 18355 \\
\hline Loudetia flammida C.E. Hubbard & $1,2,3,4$ & Erv & $\mathrm{x}$ & $\mathrm{x}$ & & 20624 \\
\hline Loudetiopsis chrysothrix Conert. & $1,3,4$ & Erv & $\mathrm{x}$ & & & 18982 \\
\hline Melinis minutiflora Beauv. & $1,2,3,4$ & Erv & $\mathrm{x}$ & & & 20776 \\
\hline Otachyrium versicolor (Doell.) Henr. & $1,3,4$ & Erv & $\mathrm{x}$ & $\mathrm{x}$ & $\mathrm{x}$ & 22725 \\
\hline Panicum caaguazuense Henr. & $1,2,3$ & Erv & $\mathrm{x}$ & $\mathrm{x}$ & & 20891 \\
\hline Panicum cayennense Lam. & $1,2,3,4$ & Erv & $\mathrm{x}$ & $\mathrm{x}$ & & 22321 \\
\hline Panicum cervicatum Chase & 1 & Erv & $\mathrm{x}$ & & & 21520 \\
\hline Panicum decipiens Kunth & $1,2,3$ & Erv & $\mathrm{x}$ & $\mathrm{x}$ & & 21807 \\
\hline Panicum laxum Sw. & 1,2 & Erv & $\mathrm{x}$ & $\mathrm{x}$ & $\mathrm{x}$ & 19684 \\
\hline Paspalum cordatum Hackel & 4 & Erv & & $\mathrm{x}$ & $\mathrm{x}$ & 22262 \\
\hline Paspalum flaccidum Nees * & 1,2 & Erv & $\mathrm{x}$ & & & 21341 \\
\hline Paspalum gardnerianum Nees & $1,3,4$ & Erv & $\mathrm{x}$ & $\mathrm{x}$ & & 20438 \\
\hline Paspalum hyalinum Nees ex Trin. & 1 & Erv & $\mathrm{x}$ & & & 23544 \\
\hline Paspalum lineare Fourn. ex Hemsl. & $1,2,3,4$ & Erv & $\mathrm{x}$ & $\mathrm{x}$ & $\mathrm{x}$ & 21563 \\
\hline Paspalum pectinatum Nees & 1 & Erv & $\mathrm{x}$ & & & 17237 \\
\hline Paspalum sp.1 & $1,2,4$ & Erv & $\mathrm{x}$ & & & 18350 \\
\hline Paspalum sp.2 & 1 & Erv & & $\mathrm{x}$ & & 17960 \\
\hline Paspalum sp.3 & 2 & Erv & $\mathrm{x}$ & $\mathrm{x}$ & & 22761 \\
\hline Saccharum cf. asperum Steud. & 2 & Erv & & $\mathrm{x}$ & $\mathrm{x}$ & 20782 \\
\hline Schizachyrium condensatum Nees & $1,2,3,4$ & Erv & $\mathrm{x}$ & $\mathrm{x}$ & & 20775 \\
\hline Schizachyrium sp.1 & $1,2,3,4$ & Erv & $\mathrm{x}$ & $\mathrm{x}$ & & 19051 \\
\hline Schizachyrium sp.2 & $1,2,3$ & Erv & $\mathrm{x}$ & $\mathrm{x}$ & & 22575 \\
\hline Schizachyrium tenereum Nees & 1,2 & Erv & & $\mathrm{x}$ & & 22529 \\
\hline
\end{tabular}


(cont.)

\begin{tabular}{|c|c|c|c|c|c|c|}
\hline Famílias / espécies & Veredas & Hábito & B & $\mathrm{M}$ & $\mathrm{F}$ & $\mathrm{N}$ \\
\hline \multicolumn{7}{|l|}{ POACEAE } \\
\hline Setaria punicifolia (Morong.) Lind. * & $2,3,4$ & Erv & & $\mathrm{x}$ & $\mathrm{x}$ & 19097 \\
\hline Setaria sp. & 2 & Erv & $\mathrm{x}$ & $\mathrm{x}$ & & 22356 \\
\hline Sorghastrum pellitum Parodi & $2,3,4$ & Erv & $\mathrm{x}$ & $\mathrm{x}$ & & 21367 \\
\hline Sorghum halepense (L.) Pers. & 2 & Erv & $\mathrm{x}$ & & & 19047 \\
\hline Sporobolus reflexus S.C.Boechat \& H.M. Longhi-Wagner & 1 & Erv & $\mathrm{x}$ & $\mathrm{x}$ & & 21591 \\
\hline Trachypogon spicatus (L.f.) Kuntze & 1 & Erv & $\mathrm{x}$ & & & 18974 \\
\hline \multicolumn{7}{|l|}{ POLYGALACEAE } \\
\hline Polygala bracteata Benn. & 1,2 & Erv & $\mathrm{x}$ & $\mathrm{x}$ & & 20157 \\
\hline Polygala cuspidata DC. & 1 & Erv & $\mathrm{x}$ & $\mathrm{x}$ & $\mathrm{x}$ & 20082 \\
\hline Polygala heblecada Benn. & 3 & Erv & & $\mathrm{x}$ & & 18372 \\
\hline Polygala longicaulis H.B.K. & $1,2,3,4$ & Erv & $\mathrm{x}$ & $\mathrm{x}$ & & 18772 \\
\hline Polygala micropora Blake & 1 & Erv & $\mathrm{x}$ & & & 20029 \\
\hline Polygala paniculata $\mathrm{L}$. & $1,2,3,4$ & Erv & & $\mathrm{x}$ & & 22628 \\
\hline Polygala sp. & 3 & Erv & $\mathrm{x}$ & $\mathrm{x}$ & & 17301 \\
\hline Polygala timoutoides Chodat & $1,2,3$ & Erv & $\mathrm{x}$ & $\mathrm{x}$ & & 22226 \\
\hline \multicolumn{7}{|l|}{ POLYGONACEAE } \\
\hline Coccoloba cf. mollis Casar. & 3 & Arv & $\mathrm{x}$ & & & 21087 \\
\hline Polygonum persicarium St. Lag. & 2 & Erv & & $\mathrm{x}$ & $\mathrm{x}$ & 18193 \\
\hline \multicolumn{7}{|l|}{ PRIMULACEAE } \\
\hline Centunculus pentandrus R. Br. & 2 & Sub & & & $\mathrm{x}$ & 19675 \\
\hline \multicolumn{7}{|l|}{ PROTEACEAE } \\
\hline Euplassa inaequalis (Pohl) Engl. & 1,3 & Arv & $\mathrm{x}$ & & $\mathrm{x}$ & 20842 \\
\hline \multicolumn{7}{|l|}{ PTERIDACEAE } \\
\hline Adiantum serratodentatum Willd. & 3 & Erv & & & $\mathrm{x}$ & 19708 \\
\hline Ptyrograma calomelanos (L.) Link & 4 & Erv & & & $\mathrm{x}$ & 19397 \\
\hline \multicolumn{7}{|l|}{ RAPATEACEAE } \\
\hline Cephalostemon angustatus Malme & 1,2 & Erv & & $\mathrm{x}$ & $\mathrm{x}$ & 18897 \\
\hline \multicolumn{7}{|l|}{ RUBIACEAE } \\
\hline Alibertia myrciifolia K. Schum. & 4 & Sub & $\mathrm{x}$ & & & 21866 \\
\hline Borreria aff. hispida K. Schum. & 2 & Erv & $\mathrm{x}$ & $\mathrm{x}$ & & 18792 \\
\hline Borreria poaya (A. St.-Hil.) DC. & $1,2,3,4$ & Erv & $\mathrm{x}$ & $\mathrm{x}$ & & 21820 \\
\hline Borreria suaveolens G.F.W. Meyer & 1,4 & Sub & $\mathrm{x}$ & & & 22480 \\
\hline Borreria verticillata G.F.W. Meyer & $1,3,4$ & Erv & $\mathrm{x}$ & & & 23490 \\
\hline Coccocypselum sp. & 1 & Erv & & & $\mathrm{x}$ & 18782 \\
\hline Declieuxia fruticosa (Wild. ex Ruiz \& Pav.) Kuntze & 1,4 & Sub & $\mathrm{x}$ & & & 22303 \\
\hline Galianthe eupatorioides (Cham. \& Schlecht.) E.L. Cabral & $1,3,4$ & Erv & & & $\mathrm{x}$ & 22872 \\
\hline Palicourea rigida Kunth. & 1 & Arb & $\mathrm{x}$ & & & 22634 \\
\hline Perama hirsuta Aubl. & 1 & Erv & $\mathrm{x}$ & $\mathrm{x}$ & & 22036 \\
\hline Posoqueria cf. latifolia (Rudge) Roem. \& Schult. & 1 & Arv & & & $\mathrm{x}$ & 21204 \\
\hline Psychotria cf. prunifolia (H.B.K) Steyerm. & 1 & Sub & & & $\mathrm{x}$ & 18784 \\
\hline Psychotria paracatuensis Standley & 1,3 & Arb & & & $\mathrm{x}$ & 22671 \\
\hline Richardia grandiflora (Cham. \& Schlecht.) Steud. & 1 & Erv & $\mathrm{x}$ & & & 21402 \\
\hline Rudgea viburnoides (Cham.) Benth. & 3,4 & Arv & $\mathrm{x}$ & & & 17813 \\
\hline Sipania pratensis Aubl. & 3,4 & Sub & $\mathrm{x}$ & $\mathrm{x}$ & & 22682 \\
\hline Spermacoce capitata Sessé \& Moc. & 2 & Erv & $\mathrm{x}$ & & & 22227 \\
\hline \multicolumn{7}{|l|}{ SAPINDACEAE } \\
\hline Matayba guianensis Aubl. & 1,2 & Arv & $\mathrm{x}$ & & & 21763 \\
\hline Serjania erecta Radlk. & 3 & Lia & & $\mathrm{x}$ & & 21506 \\
\hline \multicolumn{7}{|l|}{ SCROPHULARIACEAE } \\
\hline Alectra stricta Benth. & 1 & & $\mathrm{x}$ & & & 23035 \\
\hline Angelonia sp. & 1 & Erv & & & $\mathrm{x}$ & 19911 \\
\hline
\end{tabular}


(cont.)

\begin{tabular}{|c|c|c|c|c|c|c|}
\hline Famílias / espécies & Veredas & Hábito & B & $\mathrm{M}$ & $\mathrm{F}$ & $\mathrm{N}$ \\
\hline \multicolumn{7}{|l|}{ SCROPHULARIACEAE } \\
\hline Bacopa salzmanni Chod. \& Hassl. & 2 & Sub & & $\mathrm{x}$ & & 18331 \\
\hline Bacopa scabra (Benth.) Descole \& Borsini & 4 & Sub & $\mathrm{x}$ & & & 17363 \\
\hline Bacopa sp. & 2 & Sub & & & $\mathrm{x}$ & 21758 \\
\hline Buchnera elongata Sw. & $1,2,3,4$ & Sub & $\mathrm{x}$ & $\mathrm{x}$ & & 18795 \\
\hline Buchnera juncea Cham. \& Schlecht. & $1,2,3,4$ & Sub & $\mathrm{x}$ & $\mathrm{x}$ & & 17300 \\
\hline Esterhazya macrodonta Cham. \& Schlecht. & 1 & Sub & $\mathrm{x}$ & $\mathrm{x}$ & & 19413 \\
\hline Esterhazya splendida Mikan & 1 & Sub & & $\mathrm{x}$ & & 20902 \\
\hline Scoparia dulcis L. & 4 & Sub & $\mathrm{x}$ & & & 22331 \\
\hline \multicolumn{7}{|l|}{ SOLANACEAE } \\
\hline Brunfelsia obovata Benth. & 1 & Arb & & $\mathrm{x}$ & $\mathrm{x}$ & 19831 \\
\hline Cestrum aff. megalophyllum Dunal & 2 & Arb & & & $\mathrm{x}$ & 18327 \\
\hline Cestrum schlechtendalii G. Don & 1,3 & Arb & & & $\mathrm{x}$ & 20062 \\
\hline Schwenkia sp. & 1 & Sub & $\mathrm{x}$ & & & 22777 \\
\hline Solanum cf. americanum Mill. & $1,2,4$ & Arb & & $\mathrm{x}$ & & 20051 \\
\hline Solanum lycocarpum A. St.-Hil. & 3,4 & Arb & $\mathrm{x}$ & $\mathrm{x}$ & & 21507 \\
\hline Solanum paniculatum L. & 4 & Arb & & & $\mathrm{x}$ & 20183 \\
\hline \multicolumn{7}{|l|}{ STERCULIACEAE } \\
\hline Byttneria oblonga Pohl & $1,2,3,4$ & Sub & $\mathrm{x}$ & $\mathrm{x}$ & $\mathrm{x}$ & 21508 \\
\hline Melochia cf. parvifolia H.B.K. & 2 & Sub & & & $\mathrm{x}$ & 21655 \\
\hline Melochia pilosa (Mill.) Fawc. \& Rendle & 2 & Erv & & $\mathrm{x}$ & & 18205 \\
\hline Melochia villosa (Mill.) Fawc. \& Rendle & 1 & Sub & $\mathrm{x}$ & & & 21346 \\
\hline Waltheria communis A. St.-Hil. & 1 & Sub & $\mathrm{x}$ & & & 17026 \\
\hline \multicolumn{7}{|l|}{ STYRACACEAE } \\
\hline Styrax ferrugineus Nees \& Mart. & 3,4 & Arv & $\mathrm{x}$ & & & 20408 \\
\hline \multicolumn{7}{|l|}{ SYMPLOCACEAE } \\
\hline Symplocos nitens (Pohl) Benth. & 4 & Arv & $\mathrm{x}$ & & & 21862 \\
\hline \multicolumn{7}{|l|}{ THELYPTERIDACEAE } \\
\hline Thelypteris salzmannii (Fee) Morton & 1 & Erv & & $\mathrm{x}$ & & 23498 \\
\hline \multicolumn{7}{|l|}{ TILIACEAE } \\
\hline Triumfetta sp. & $1,2,4$ & Sub & & $\mathrm{x}$ & & 23505 \\
\hline \multicolumn{7}{|l|}{ ULMACEAE } \\
\hline Trema micrantha (L.) Blume & 4 & Arv & & & $\mathrm{x}$ & 21293 \\
\hline \multicolumn{7}{|l|}{ VERBENACEAE } \\
\hline Casselia sp. & 2 & Sub & $\mathrm{x}$ & $\mathrm{x}$ & & 18329 \\
\hline Lippia cf. primulina $\mathrm{S}$. Moore & 2 & Sub & $\mathrm{x}$ & & & 21398 \\
\hline Lippia cf. salviaefolia Cham. & 1 & Sub & $\mathrm{x}$ & & & 19805 \\
\hline Lippia corymbosa Cham. & 3 & Sub & $\mathrm{x}$ & & & 19865 \\
\hline Lippia lupulina Cham. & 1 & Sub & $\mathrm{x}$ & & & 21355 \\
\hline Stachytarpheta sanguinea Mart. & 2 & Sub & $\mathrm{x}$ & & & 19703 \\
\hline \multicolumn{7}{|l|}{ VITACEAE } \\
\hline Cissus duarteana Cambess. & 4 & Lia & & & $\mathrm{x}$ & 19415 \\
\hline \multicolumn{7}{|l|}{ VOCHYSIACEAE } \\
\hline Qualea multiflora Mart. & 1 & Arv & $\mathrm{x}$ & & & 18220 \\
\hline Vochysia tucanorum Mart. & 4 & Arv & $\mathrm{x}$ & & & 18804 \\
\hline \multicolumn{7}{|l|}{ XYRIDACEAE } \\
\hline Abolboda puchella Humb. \& Bonpl. & $1,2,4$ & Erv & $\mathrm{x}$ & $\mathrm{x}$ & $\mathrm{x}$ & 19704 \\
\hline Abolboda sp. & $2,3,4$ & Erv & $\mathrm{x}$ & $\mathrm{x}$ & & 18826 \\
\hline Xyris asperula Mart. & $1,2,3$ & Erv & $\mathrm{x}$ & $\mathrm{x}$ & & 22197 \\
\hline Xyris cf. goyazensis Malme & 1 & Erv & $\mathrm{x}$ & $\mathrm{x}$ & & 17781 \\
\hline Xyris cf. schizachne Mart. & 2 & Erv & & $\mathrm{x}$ & & 18829 \\
\hline Xyris jupicai Rich. & $1,2,3,4$ & Erv & $\mathrm{x}$ & $\mathrm{x}$ & $\mathrm{x}$ & 20120 \\
\hline
\end{tabular}


(cont.)

\begin{tabular}{|c|c|c|c|c|c|c|}
\hline Famílias / espécies & Veredas & Hábito & B & $\mathrm{M}$ & $\mathrm{F}$ & $\mathrm{N}$ \\
\hline \multicolumn{7}{|l|}{ XYRIDACEAE } \\
\hline Xyris savanensis Miq. & $1,2,3,4$ & Erv & $\mathrm{x}$ & $\mathrm{x}$ & $\mathrm{x}$ & 18808 \\
\hline Xyris sp. $1 *$ & 3 & Erv & $\mathrm{x}$ & $\mathrm{x}$ & & 21475 \\
\hline Xyris sp.2 & $1,2,3$ & Erv & $\mathrm{x}$ & $\mathrm{x}$ & & 21826 \\
\hline Xyris sp.3 & 1,4 & Erv & $\mathrm{x}$ & $\mathrm{x}$ & & 19786 \\
\hline Xyris tenella Kunth & $1,2,3,4$ & Erv & $\mathrm{x}$ & $\mathrm{x}$ & $\mathrm{x}$ & 21354 \\
\hline Xyris tortula Mart. * & $1,2,3,4$ & Erv & $\mathrm{x}$ & $\mathrm{x}$ & $\mathrm{x}$ & 20421 \\
\hline \multicolumn{7}{|l|}{ ZINGIBERACEAE } \\
\hline Costus spiralis (Jacq.) Roscoe & 1,3 & Erv & & & $\mathrm{x}$ & 22668 \\
\hline
\end{tabular}

*=espécies com folhas fililiformes

sendo que $25 \%$ do total ocorreram exclusivamente nessa área (tabela 1), onde houve predomínio das espécies herbáceas e subarbustivas (87,9\%) como: Echinodorus latifolius, Barjonia harleyi, Chaptalia integrifolia, Elephantopus palustris, Eupatorium amigdalinum e Burmannia flava. Por outro lado, observou-se ausência do elemento arbóreo e apenas $7 \%$ das espécies presentes foram arbustivas.

$\mathrm{Na}$ zona de fundo ocorreu a menor riqueza florística (136 espécies), sendo que 38,2\% foram exclusivas. Nessa zona, $73,4 \%$ das espécies pertencem ao estrato herbáceo-subarbustivo. As arbustivas e as arbóreas, juntas, constituiram $18,4 \%$ das espécies dessa área (tabela 1). Tapirira guianensis, Cecropia pachystachya, Miconia chamissois, Miconia theaezans e Ilex affinis foram encontradas formando moitas sob os adultos da palmeira buriti. Porém, em geral, no fundo da vereda predomina o hábito herbáceo, principalmente com espécies das famílias Cyperaceae, Poaceae e

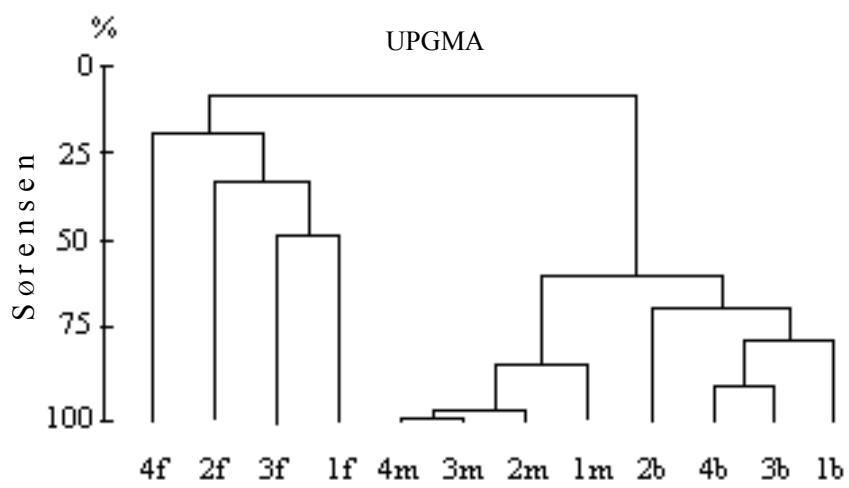

Figura 2. Análise de agrupamento das floras amostradas nas zonas de fundo (f), meio (m) e borda (b) das quatro veredas situadas no Município de Uberlândia, MG. Vereda 1 (Reserva Vegetal do CCPIU), vereda 2 (Estrada para Campo Florido), veredas 3 e 4 (Estação Ecológica do Panga).
Eriocaulaceae (tabela 1).

Nas veredas estudadas encontraram-se 27 espécies herbáceas com folhas filiformes, pertencentes às famílias Cyperaceae, Poaceae e Xyridaceae (tabela 1). Setaria punicifolia (Poaceae) e Rhynchospora spp. (Cyperaceae) formam densas touceiras, constituídas por um emaranhado de folhas vivas e mortas. Nas aberturas entre as touceiras foram amostradas algumas espécies de Eriocaulaceae exclusivas desse ambiente como: Eriocaulon sp., Paepalanthus speciosus e P. cachambuensis.

\section{Discussão}

A grande riqueza florística encontrada nas veredas amostradas no Município de Uberlândia deve-se, possivelmente, à existência de diferentes ambientes edáficos sob essas comunidades vegetais, especialmente devido às condições de umidade. A ocorrência de um gradiente de umidade da borda (solo mais seco, próximo ao cerrado) para o fundo da vereda (solo mais úmido) foi constatada na Estação Ecológica do Panga por Guimarães (2001) e na Reserva Vegetal do Clube Caça e Pesca por Amaral (2002). Esse fato pode ser o responsável pela zonação das espécies vegetais (borda, meio e fundo) comprovada na análise de agrupamento do presente levantamento florístico.

O hábito herbáceo-subarbustivo predomina em todas as zonas das veredas amostradas conferindo-lhes uma fisionomia predominantemente campestre com limites bem definidos com a vegetação arbustivo-arbórea do cerrado. A dominância do estrato herbáceo-subarbustivo deve-se à riqueza de espécies de Asteraceae, Cyperaceae e Poaceae que, de acordo com Coutinho (1978), reúnem gêneros e espécies com grande número de representantes heliófilos. 
Em geral, várias espécies que foram coletadas nessas veredas não foram restritas a estas fitocenoses, sendo também encontradas em outras formações campestres, savânicas e florestais. As duas famílias mais ricas amostradas aqui (Poaceae e Asteraceae) também tiveram alta riqueza em outras comunidades campestres em Minas Gerais (Andrade et al. 1986), no Rio Grande do Sul (Zocche \& Porto 1992, Boldrini \& Eggers 1996, Boldrini et al. 1998) e no componente campestre de cerrado no Estado de São Paulo (Mantovani \& Martins 1993).

Um total de 20,6\% de espécies de Asteraceae e $9,4 \%$ de Poaceae, foram consideradas como invasoras na flora do cerrado do Distrito Federal (Mendonça et al. 1998) e algumas delas foram citadas também na flora da Reserva Pé-de-Gigante, Estado de São Paulo (Batalha \& Mantovani 2001). Essa ocorrência tem sido atribuída ao efeito da fragmentação e ao efeito de borda (Pivello et al. 1999, Batalha \& Mantovani 2001). Na vereda tais espécies foram amostradas principalmente na borda, área mais sujeita à antropização que vem ocorrendo na região (Guimarães 2001).

Uma semelhança encontrada entre a flora das veredas amostradas e fisionomias campestres refere-se à presença de espécies com folhas filiformes. Algumas espécies de Poaceae, que apresentam essa característica, por exemplo, Anthaenantiopsis trachystachya, Ctenium brevispicatum, Paspalum flaccidum e Setaria punicifolia, foram também listadas na composição florística de brejos no Distrito Federal (Mendonça et al. 1998). Outras, como Axonopus brasiliensis e Leptocoripium lanatum, que ocorreram na borda e meio da vereda, foram encontradas tanto em áreas de brejo (Pereira et al. 1985, Mendonça et al. 1998) como em campos rupestres (Andrade et al. 1986, Stanard 1995). $\mathrm{Na}$ família Cyperaceae, Rhynchospora globosa e $R$. tenuis ocorreram em áreas de campo (Boldrini et al. 1998, Zocche \& Porto 1992) enquanto Eleocharis capillacea foi listada para campo rupestre (Stanard 1995). O mesmo foi observado para duas espécies de Xyridaceae (Xyris tortula e Xyris sp.1). Espécies com tais características foliares ocupam as zonas de meio e fundo formando um emaranhado denso e, possivelmente, possuem alguma vantagem competitiva na ocupação desses ambientes. No entanto, seriam necessários estudos mais detalhados para investigar melhor esta hipótese.

As densas touceiras de espécies filiformes podem estar dificultando o estabelecimento de outras espécies nas zonas mais úmidas das veredas. Foi observado que nessas condições algumas espécies das famílias
Lamiaceae (Hyptis spp., Peltodon spp.), Scrophulariaceae (Buchnera spp.), Acanthaceae (Justicia spp.), Ochnaceae (Sauvagesia racemosa), Apocynaceae (Rhabdadenia sp.) e Rapateaceae (Cephalostemon angustatus) conseguem se estabelecer apresentando alongamento caulinar transpondo as densas touceiras com folhas filiformes e expondo suas inflorescências e infrutescências.

Caesalpiniaceae e Fabaceae, conhecidas por seu grande número de espécies arbustivas e arbóreas nos cerrados (Goodland 1970, Mantovani \& Martins 1993, Castro et al. 1999), ocorreram geralmente na borda das veredas onde o ambiente assemelha-se aos campos de cerrado da região. No entanto, as espécies coletadas apresentaram principalmente hábito subarbustivo demonstrando que o ambiente parece ser limitante para o estabelecimento de outras formas de vida de maior porte.

Eriocaulaceae e Xyridaceae ocorrem em áreas abertas e brejosas (Joly 1979), e em campos rupestres (Pirani et al. 1994). Nas veredas estudadas, ocorrem em áreas mais úmidas notando-se poucos indivíduos para a maioria das espécies. Syngonanthus (Eriocaulaceae) e Xyris (Xyridaceae), gêneros com maior número de espécies, foram encontrados nas mesmas condições no Distrito Federal (Pereira et al. 1985, Mendonça et al. 1998) e em baixios de campos rupestres (Pirani et al. 1994, Stanard 1995). Espécies pertencentes a esses gêneros, como: Xyris savanensis, Syngonanthus caulescens e $S$. nitens, são encontrados nas fitocenoses acima referidas, destacando-se mais uma vez que a distribuição de algumas espécies encontradas na vereda não está restrita a esse ambiente.

Dentre as famílias com uma única espécie, 25 ocorreram nos ambientes mais úmidos da vereda (meio e fundo). Destas, Mayacaceae e Hydrocaritaceae são aquáticas, Rapateaceae, Droseraceae, Alismataceae, Burmanniaceae e Hydrophylaceae vivem em terrenos brejosos e as demais podem ocorrer em ambientes mais secos (Joly 1979). Isso demonstra que grande número de famílias com apenas uma espécie, são restritas aos ambientes mais úmidos sendo, possivelmente, as espécies mais sensíveis aos processos de antropização.

As espécies arbóreas, foram encontradas, em sua maioria, no limite da vereda com o cerrado, em solo mais seco. Na borda da vereda o lençol freático varia de $0,7 \mathrm{~m}$, de profundidade na estação chuvosa, a 2,5 m na estação relativamente seca (Guimarães 2001, Amaral 2002). Nessa área de transição ocorreram Miconia albicans (Melastomataceae), Styrax ferrugineus 
(Styracaceae), Vochysia tucanorum (Vochysiaceae), Blepharocalyx salicifolius, Myrcia rostrata e Myrcia tomentosa (Myrtaceae). Estas espécies não são exclusivas nas bordas das veredas, sendo também citadas para outras fisionomias do cerrado do Triângulo Mineiro (Goodland 1970, Araújo et al. 1997). Por outro lado, outras arbóreas como Tapirira guianensis, Cecropia pachystachya, Hedyosmum brasiliense e Piper regnellii, amostradas no fundo da vereda, foram também relacionadas para as matas galerias do Distrito Federal por Pereira et al. (1985) e Mendonça et al. (1998). Segundo Carvalho (1991), a presença de epécies de mata de galeria em áreas de veredas pode sugerir mudanças em seu estádio sucessional devido ao constante assoreamento e modificações na rede de drenagem.

As veredas estudadas apresentaram alto número de espécies distribuídas em um gradiente de umidade do solo. As maiores afinidades florísticas entre as zonas de borda e meio da vereda pode sugerir que esses ambientes sejam mais uniformes quanto às características edáficas. Por outro lado, as comunidades de fundo apresentaram menor afinidade entre si, possivelmente devido às diferenças na rede de drenagem entre as veredas.

A presença de vários táxons com folhas filiformes juntamente com a palmeira buriti, caracterizaram as veredas estudadas. A riqueza florística aliada à zonação de espécies permite sugerir que a vereda seja considerada como um "complexo vegetacional". Possivelmente, algumas espécies, a exemplo do buriti, sejam exclusivas desse ambiente. Entretanto para a comprovação dessas idéias é necessário que levantamentos florísticos sejam feitos em outras veredas, incluindo estudos de características edáficas e ecológicas. Discussões mais refinadas a esse respeito não puderam ser feitas devido ao número restrito de informação disponível na literatura sobre essa fitocenose.

Agradecimentos - À Fapemig pelo financiamento do projeto (Processo 231597). Aos seguintes especialistas pela identificação de espécies das famílias: Melastomataceae (Dra. Rosana Romero e Dra. Cássia B.R. Munhoz); Asteraceae (Dr. Jimi Naoki Nakajima); Poaceae (Dr. Tarcisio S. Filgueiras, Dra. Regina Célia Oliveira e Dr. José F.M. Valls); Cyperaceae (Dr. Fábio Vitta, MS. Ana Cláudia Araújo); Myrtaceae (Dra. Adriana Assis Arantes); Orchidaceae (Dr. João A.N. Batista) e Eriocaulaceae (Dra. Nancy Hensold). Ao técnico Péricles Andrade Faria pelo auxílio nos trabalhos de campo e na preparação do material para a inclusão no herbário.

\section{Referências bibliográficas}

ACHÁ-PANOSO, L. 1978. Levantamento detalhado dos solos da área sob a influência do Reservatório de Três Marias, MG. Embrapa/Epamig. Boletim Técnico 57:22-29.

ALMEIDA, J.R., BARUQUI, F.M., BARUQUI, A.M. \& MOTTA, P.E.F. 1983. Principais solos de várzeas do Estado de Minas Gerais e suas potencialidades agrícolas. Informe Agropecuário 9:70-78.

AMARAL, A.F. 2002. Caracterização fenológica, e aspectos do solo em áreas queimada e desbastada de uma vereda em Uberlândia, MG. Dissertação de mestrado, Universidade Federal de Uberlândia, Uberlândia.

ANDRADE, P.M., GONTIJO, T.A. \& GRANDI, T.S.M. 1986. Composição florística e aspectos estruturais de uma área de "campo rupestre" do morro do Chapéu, Nova Lima, Minas Gerais. Revista Brasileira de Botânica 9:13-21.

ARAÚJO, G.M., NUNES, J.J., ROSA, A.G. \& RESENDE, E.J. 1997. Estrutura comunitária de vinte áreas de cerrados residuais no Município de Uberlândia, MG. Daphne 7:7-14.

BATALHA, M.A. \& MANTOVANI, W. 2001. Floristic composition of the cerrado in the Pé-de-Gigante Reserve (Santa Rita do Passa Quatro, southeastern Brazil). Acta Botanica Brasilica 15:289-304.

BOAVENTURA, R.S. 1978. Estudo das veredas da Serra do Cabral. Cetec, Belo Horizonte.

BOLDRINI, I.I., EGGERS, L. 1996. Vegetação campestre do Sul do Brasil: dinâmica de espécies à exclusão do gado. Acta Botanica Brasilica 10:37-58.

BOLDRINI, I.I., MIOTTO, S.T.S., LONGHI-WAGNER, H.M., PILLAR, V.P. \& MARZALL, K. 1998. Aspectos florísticos e ecológicos da vegetação campestre do Morro da Polícia, Porto Alegre, RS. Acta Botanica Brasilica 12:89-100.

CARVALHO, P.G.S. 1991. As veredas e sua importância no domínio dos cerrados. Informe Agropecuário 168:47-54.

CASTRO, A.A.J.F., MARTINS, F.R., TAMASHIRO, J.Y. \& SHEPHERD, G.J. 1999. How rich is the flora of Brazilian cerrados? Annals of the Missouri Botanical Garden 86:192-244.

CASTRO, J.P.C. 1980. As veredas e a sua proteção jurídica. Análise e Conjuntura 10:321-331.

COUTO, E.G., RESENDE, M.B. \& REZENDE, S.B. 1985. Terra ardendo. Ciência Hoje 16:48-57.

COUTINHO, L.M. 1978. O conceito de Cerrado. Revista Brasileira de Botânica 1:17-23.

CRONQUIST, A. 1988. The evolution and classification of flowering plants. Bronx: New York Botanical Garden, New York.

CURI, P.R. 1982. Análise de agrupamento: métodos seqüenciais, aglomerativos e hierárquicos. Ciência e Cultura 35:1416-1429.

EITEN, G. 1983. Classificação da vegetação do Brasil. CNPq, Brasília. 
EITEN, G. 1994. Vegetação. In Cerrado: caracterização, ocupação e perspectivas (M.N. Pinto, org.). Editora da Universidade de Brasília, Brasília, p.17-73.

EMBRAPA. 1982. Levantamento de reconhecimento de média intensidade dos solos e avaliação da aptidão agrícola das terras do Triângulo Mineiro. Serviço nacional de levantamento e conservação de solos. Epamig - DRNR, Rio de Janeiro.

FELFILI, J.M., SEVILHA, A.C. \& SILVA JÚNIOR, M.C. 2001. Comparação entre as unidades fisiográficas Chapada Pratinha, Veadeiros e EspigãoMestre do São Francisco. In Biogeografia do bioma Cerrado: estudo fitofisionômico da Chapada do Espigão Mestre do São Francisco (J.M. Felfili \& M.C. Silva Júnior, orgs.). UnB, Brasília, p.80-152.

FERREIRA, M.B. 1980. O cerrado em Minas Gerais gradações e composição florística. Informe Agropecuário 61:4-8.

GOODLAND, R.J.A. 1970. Plants of the cerrado vegetation of Brasil. Phytologia 20:57-78.

GUIMARÃES, A.J.M. 2001 Características do solo e da comunidade vegetal em área natural e antropizada de uma vereda na região de Uberlândia-MG. Dissertação de mestrado, Universidade Federal de Uberlândia, Uberlândia.

JOLY, A.B. 1979. Botânica, introdução à taxonomia vegetal. Companhia Editora Nacional, São Paulo.

LIMA, S.C. 1996. As veredas do Ribeirão do Panga no Triângulo Mineiro e a evolução da paisagem. Tese de doutorado, Universidade de São Paulo, São Paulo.

LIMA, S.C. \& QUEIROZ NETO, J.P. 1996. As veredas e a evolução do relevo. Sociedade e Natureza 15:481-488.

MAcCUNE, B. \& MEFFORD, M.J. 1995. PC-ORD. Multivariate analysis of ecological data, version 2.0. MjM Software Design, Gleneden Beach, Oregon.

MAGALHÃES, G.M. 1966. Sobre os cerrados de Minas Gerais. Anais da Academia Brasileira de Ciências 38:59-69.

MAGURRAN, A.E. 1988. Ecological diversity and its measurement. Princeton University, Princeton.
MANTOVANI, W. \& MARTINS, F.R. 1993. Florística do cerrado na Reserva Biológica de Moji Guaçu, SP. Acta Botanica Brasilica 7:33-60.

MENDONÇA, R.C., FELFILI, J.M., TELES WALTER, B.M., SILVA JUNIOR, M.C., REZENDE, A.V., FILGUEIRAS, T.S. \& NOGUEIRA, P.E. 1998. Flora vascular do cerrado. In Cerrado ambiente e flora (S. Sano \& S.P. Almeida, eds). Embrapa-CPAC, Brasília, p.289-556.

PEREIRA, B.A.S., MENDONÇA, R.C., FILGUEIRAS, T.S., PAULA, J.E. \& HERINGER, E.P. 1985. Levantamento florístico da área de proteção ambiental (APA) da bacia do rio São Bartolomeu, Distrito Federal. In Anais do XXXVI Congresso Brasileiro de Botânica, (R.J.M. Oliveira, ed.). UFPR, Curitiba, p.419-492.

PIVELlO, V.R., CARVAlHO, V.M.C., LOPES, P.F., PECCININI, A.A. \& ROSSO, S. 1999. Abundance and distribution of native and alien grasses in a "cerrado" (Brazilian savanna) Biological Reserve. Biotropica 31:71-82.

PIRANI, J.R., GIULIETTI, A.M., MELLO-SILVA, R. \& MEGURO, M. 1994. Checklist and patterns of geographic distribution of the vegetation of Serra do Ambrósio, Minas Gerais, Brazil. Revista Brasileira de Botânica 17:133-147.

SILVA JÚNIOR, M.C. \& FELFILI, J.M. 1998. A vegetação da estação ecológica de Águas Emendadas. Instituto de Ecologia e Meio Ambiente do Distrito Federal, Brasília.

STANARD, B.L. 1995. Flora of the Pico das Almas, Chapada Diamantina-Bahia, Brazil. Royal Botanic Gardens, Kew.

TRYON, R.M. \& TRYON, A.F. 1982. Ferns and allied plants: with special reference to Tropical America. Springer, New York.

ZOCCHE, J.J. \& PORTO, M.L. 1992. Florística e fitossociologia de campo natural sobre banco de carvão em áreas mineradas, Rio Grande do Sul, Brasil. Acta Botanica Brasilica 6:47-84. 